\title{
Decision-Making, Errors, and Confidence in the Brain
}

\author{
Edmund T. Rolls, ${ }^{1}$ Fabian Grabenhorst, ${ }^{2}$ and Gustavo Deco ${ }^{3}$ \\ ${ }^{1}$ Oxford Centre for Computational Neuroscience, Oxford, United Kingdom; ${ }^{2}$ Department of Experimental Psychology, University of \\ Oxford, Oxford, United Kingdom; and ${ }^{3}$ Institució Catalana de Recerca i Estudis Avançats (ICREA), Universitat Pompeu Fabra, \\ Computational Neuroscience, Barcelona, Spain
}

Submitted 25 June 2010; accepted in final form 31 August 2010

\begin{abstract}
Rolls ET, Grabenhorst F, Deco G. Decision-making, errors, and confidence in the brain.. J Neurophysiol 104: 2359-2374, 2010. First published September 1, 2010; doi:10.1152/jn.00571.2010. To provide a fundamental basis for understanding decision-making and decision confidence, we analyze a neuronal spiking attractor-based model of decision-making. The model predicts probabilistic decision-making with larger neuronal responses and larger functional magnetic resonance imaging (fMRI) blood-oxygen-level-dependent (BOLD) responses on correct than on error trials because the spiking noiseinfluenced decision attractor state of the network is consistent with the external evidence. Moreover, the model predicts that the neuronal activity and the BOLD response will become larger on correct trials as the discriminability $\Delta I$ increases and confidence increases and will become smaller as confidence decreases on error trials as $\Delta I$ increases. Confidence is thus an emergent property of the model. In an fMRI study of an olfactory decision-making task, we confirm these predictions for cortical areas including medial prefrontal cortex and the cingulate cortex implicated in choice decision-making, showing a linear increase in the BOLD signal with $\Delta I$ on correct trials, and a linear decrease on error trials. These effects were not found in a control area, the orbitofrontal cortex, where reward value useful for the choice is represented on a continuous scale but that is not implicated in the choice itself. This provides a unifying approach to decision-making and decision confidence and to how spiking-related noise affects choice, confidence, synaptic and neuronal activity, and fMRI signals.
\end{abstract}

\section{N T R O D U C T I O N}

Decision-making, and our confidence in the decisions we make, are important areas in neuroscience in the new field of neuroeconomics (Deco and Rolls 2006; Heekeren et al. 2004; Kable and Glimcher 2007; Kepecs et al. 2008; Kiani and Shadlen 2009; Kim and Shadlen 1999; Rolls 2008; Romo et al. 2004; Shadlen and Newsome 2001). Fundamental issues are to understand the neural processes that underlie decision-making and decision confidence and to establish the bases for neural signatures of these processes. Evidence from primate single neuron recordings shows that neuronal responses in a motion decision-making task occur earlier on easy versus difficult trials in a visual motion decision-related brain region, the posterior parietal cortex, in which neuronal activity reflects decision confidence (Kiani and Shadlen 2009). In the human dorsolateral prefrontal cortex, higher functional magnetic resonance imaging (fMRI) blood-oxygen-level-dependent (BOLD) signals can be observed on easy trials versus difficult trials in a similar decision-making task, and this has been proposed as a signature of decision-making (Heekeren et al. 2004, 2008).

Address for reprint requests and other correspondence: E. T. Rolls, Oxford Centre for Computational Neuroscience, Oxford, England (E-mail: Edmund. Rolls@oxcns.org; www.oxcns.org).
Further, it is well established that subjective decision confidence (assessed before the outcome is known) increases with discriminability, measured by the difference between the stimuli, $\Delta I$, that is, as the decision becomes easier and the percentage correct becomes better (Jonsson et al. 2005; Vickers 1979; Vickers and Packer 1982). Consistent with this, the probability that a rat will abort a trial is higher if a decision just made is incorrect (Kepecs et al. 2008). This maps onto humans' everyday experience: if asked to judge how heavy two weights are, people are confident in their decision if one weight is much heavier than the other, and not confident if the two weights are very similar, with findings on the relation between discriminability and confidence dating back to the 19th century and reviewed by Petrusic and Baranski (2009).

Here we capitalize on recent advances in theoretical understanding of how choice decisions are made using an integrateand-fire attractor network that makes probabilistic decisions from the spontaneous firing state into one of two or more high firing rate stable attractor states each implemented by a set of coupled neurons that receives the inputs for one of the decisions and where the choice made is probabilistic because of the noise due to the random spiking times of the neurons when firing at a given rate (Deco and Rolls 2006; Deco et al. 2009; Rolls 2008; Rolls and Deco 2010; Wang 2002, 2008). We have recently shown that a network of this type predicts from its firing rates or synaptic currents a BOLD signal that increases approximately linearly with $\Delta I$ when all trials are considered and have found this BOLD signature during decisions made about the subjective pleasantness of both thermal and olfactory stimuli in the medial prefrontal cortex area 10 (Rolls et al. 2010a), a region implicated by other analyses in these pleasantness-related decisions (Grabenhorst et al. 2008; Rolls et al. 2010b).

Here we perform new analyses on how the decision-making operates on correct versus error trials. Part of the interest of this is that confidence is higher on correct than on error trials even before the outcome is known (Vickers and Packer 1982). It has been shown previously that in this integrate-and-fire decisionmaking network, in the winning attractor, the difference of the firing rates of the neurons on correct and error trials increases with $\Delta I$ (Wang 2002). In the new computational neuroscience analyses described here, we show that the firing rates of the neurons in the winning attractor increase approximately linearly as $\Delta I$ increases from 0 on correct trials and decrease approximately linearly as $\Delta I$ increases on error trials. The reason for this is that on correct trials, when the network influenced by the spiking noise has reached an attractor state supported by the recurrent connections between the neurons, the external inputs to the network that provide the evidence for 
the decision have firing rates that are consistent with the decision and increase the firing rates further and proportionally to $\Delta I$, whereas on error trials, the inputs do not support the attractor-related firing. We also show that in the losing attractor, the low firing rates decrease approximately linearly on correct trials and increase approximately linearly on error trials as $\Delta I$ increases. The explanation is analogous to that just provided, combined with effects from the competition between the winning and the losing attractor mediated through the inhibitory neurons. In the integrate-and-fire decision-making attractor network, we then use all the activity from the winning and losing neurons to predict that the net BOLD signal will increase approximately linearly with $\Delta I$ on correct trials and decrease approximately linearly with $\Delta I$ on error trials. In new functional imaging analyses, we then show that during decision making about which of two odors is more pleasant, this signature of the BOLD signal increasing as a function of $\Delta \mathrm{I}$ on correct trials, and decreasing as a function of $\Delta I$ on error trials, which is how decision confidence behaves (Vickers and Packer 1982), is found in the medial prefrontal cortex area 10, the posterior and subgenual cingulate cortex, and the dorsolateral prefrontal cortex, but not in the mid-orbitofrontal cortex, where activations instead reflect the pleasantness or subjective affective value of the stimuli used as inputs to the choice decisionmaking process.

\section{METHODS}

\section{Modeling investigations}

The theoretical framework of the model used here was introduced by Wang (2002) and developed further (Deco and Rolls 2006; Deco et al. 2007, 2009; Marti et al. 2008). In this framework, we model probabilistic decision-making by a network of interacting neurons organized into a discrete set of populations as depicted in Fig. 1. Populations or pools of neurons are defined as groups of excitatory or inhibitory neurons sharing the same inputs and connectivities. The
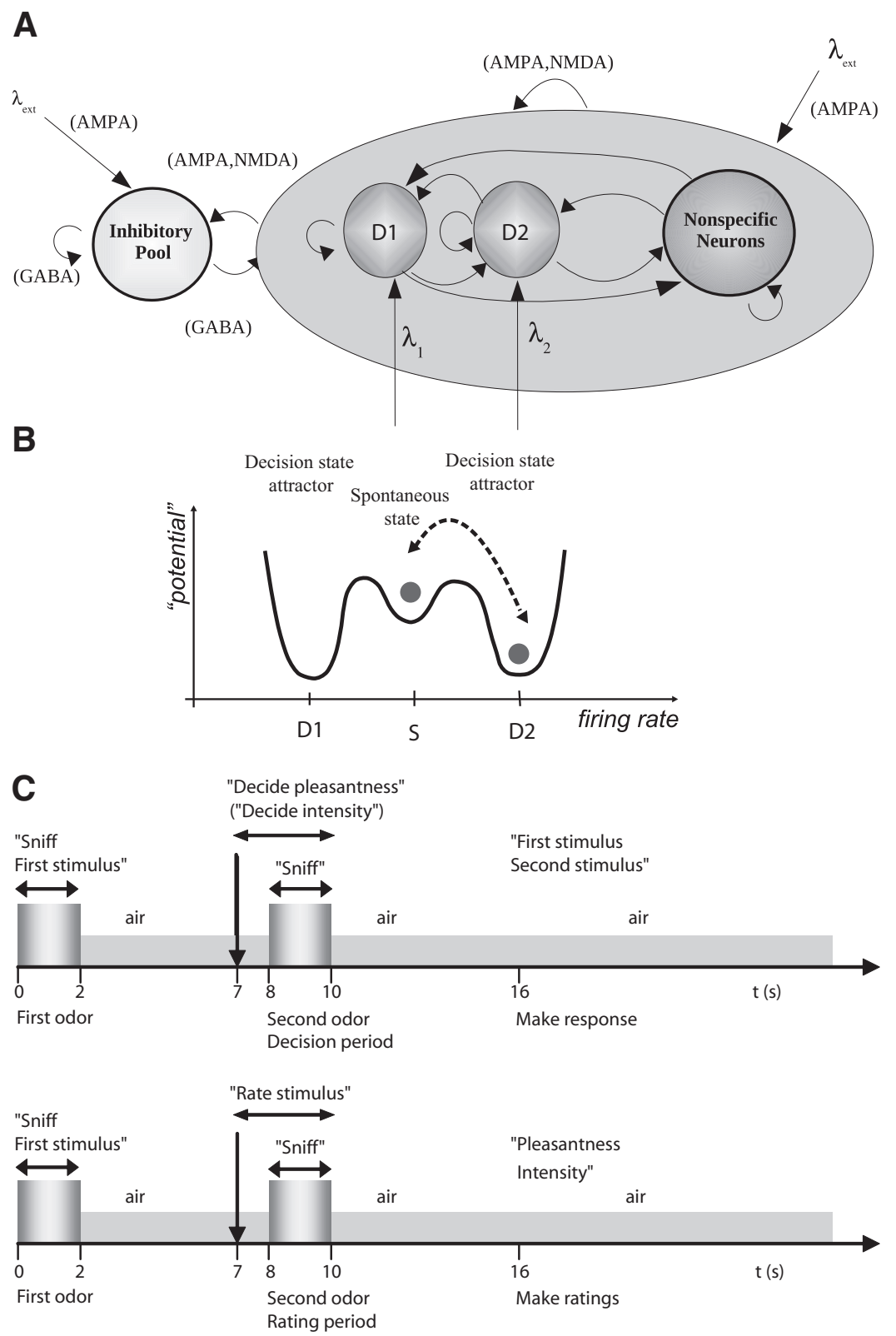

FIG. 1. A: the architecture of the integrate-and-fire attractor network used to model decision-making (see text). The evidence for decision 1 is applied via the $\lambda_{1}$ inputs to neuronal population or pool D1 and for decision 2 via the $\lambda_{2}$ inputs to pool D2. The synaptic weights $w_{\mathrm{ij}}$ have been associatively modified during training in the presence of $\lambda_{1}$ and at a different time of $\lambda_{2}$. When $\lambda_{1}$ and $\lambda_{2}$ are applied, each attractor competes through the inhibitory interneurons until one wins the competition, and the network falls into one of the high firing rate attractors that represents the decision. The noise in the network caused by the random spiking of the neurons means that on some trials, for given inputs, the neurons in the decision 1 (D1) attractor are more likely to win, and on other trials, the neurons in the decision 2 (D2) attractor are more likely to win. This makes the decision-making probabilistic, for, as shown in $B$, the noise influences when the system will jump out of the spontaneous firing stable (low energy) state $S$, and whether it jumps into the high firing state for decision 1 (D1) or decision 2 (D2). B: a multistable "effective energy landscape" for decision-making with stable states shown as low "potential" basins. Even when the inputs are being applied to the network, the spontaneous firing rate state is stable, and noise provokes transitions into the high firing rate decision attractor state D1 or D2 (see Rolls and Deco 2010). C: task design for trials of the human functional magnetic resonance imaging (fMRI) olfactory task. On decision trials (top), the task required a binary decision starting during the 2 nd odor about which of the 2 odors was more pleasant, or (on different trials, as indicated by the instruction at $t=7 \mathrm{~s}$ ) more intense. On rating trials (bottom), identical stimuli were used, but no decision was required, and instead participants rated the 2nd odor for pleasantness and intensity on continuous analog visual rating scales. The trial types were identical until $t=7 \mathrm{~s}$, when the instruction indicated whether the trial type was decide or rate. The 2 nd odor was delivered at $t=8 \mathrm{~s}$, the subjects were deciding or rating at that time, and the imaging was with respect to this period starting at $t=8 \mathrm{~s}$. No responses to indicate the decision made or the rating value could be made until $t=16 \mathrm{~s}$. Data from the pleasantness decision-making trials were analyzed here. 
network contains $N_{\mathrm{E}}$ (excitatory) pyramidal cells and $N_{\mathrm{I}}$ inhibitory interneurons. In our simulations, we use $N_{\mathrm{E}}=400$ and $N_{\mathrm{I}}=100$, consistent with the neurophysiologically observed proportion of $80 \%$ pyramidal cells versus 20\% interneurons (Abeles 1991; Rolls and Deco 2002). The neurons are fully connected (with synaptic strengths as specified in the following text). In the model, the specific populations D1 (for decision 1) and D2 encode the categorical result of the choice between the two stimuli that activate each of these populations. Each specific population of excitatory cells contains $r N_{\mathrm{E}}$ neurons (in our simulations $r=0.1$ ). In addition there is one nonspecific population, named "nonspecific," which groups all other excitatory neurons in the modeled brain area not involved in the present tasks, and one inhibitory population, named "inhibitory," grouping the local inhibitory neurons in the modeled brain area. The latter population regulates the overall activity and implements competition in the network by spreading a global inhibition signal across the network being modeled.

Because we are mainly interested in the nonstationary probabilistic behavior of the network, the proper level of description at the microscopic level is captured by the spiking and synaptic dynamics of one-compartment integrate-and-fire (IF) neuron models (Brunel and Wang 2001; Dayan and Abbott 2001; Deco and Rolls 2006; Rolls 2008; Rolls and Deco 2010). An IF neuron integrates the afferent current generated by the incoming spikes and fires when the depolarization of the cell membrane crosses a threshold. At this level of detail, the model allows the use of realistic biophysical time constants, latencies, and conductances to model the synaptic current, which in turn allows a thorough study of the realistic time scales and firing rates involved in the time evolution of the neural activity. Consequently, the simulated neuronal dynamics that putatively underlie cognitive processes can be quantitatively compared with experimental data. The IF neurons are modeled as having three types of receptor mediating the synaptic currents flowing into them: AMPA, $N$-methyl-D-aspartate (NMDA; both activated by glutamate), and GABA receptors. The excitatory recurrent postsynaptic currents (EPSCs) are considered to be mediated by AMPA (fast) and NMDA (slow) receptors; external EPSCs imposed onto the network from outside are modeled as being driven only by AMPA receptors. Inhibitory postsynaptic currents (IPSCs) to both excitatory and inhibitory neurons are mediated by GABA receptors. The details of the mathematical formulation are summarized in previous papers (Brunel and Wang 2001; Deco and Rolls 2006) and are provided in the supplementary material ${ }^{1}$ and by Rolls and Deco (2010).

We weight the conductance values for the synapses between pairs of neurons by synaptic connection weights, which can deviate from their default value, which is 1.0 unless otherwise specified. The structure and function of the network are achieved by differentially setting the synaptic weights within and between populations of neurons that are shown in Fig. 1. We assume that the connections are already formed, by for example earlier self-organization mechanisms, as if they were established by Hebbian learning, i.e., the coupling will be strong if the pair of neurons have correlated activity (i.e., covarying firing rates), and weak if they are activated in an uncorrelated way. On the basis of the mean field analysis of this network (Deco and Rolls 2006; Rolls and Deco 2010), neurons within a specific excitatory population (D1, and D2) are mutually coupled with a strong synaptic weight $w_{+}$, set to 2.1 for the simulations described here. Furthermore, the populations encoding for these two decisions are likely to have anti-correlated activity in this behavioral context, resulting in weaker than average connections between the two different populations. Consequently, we choose a weaker value $w_{-}=1-r\left(w_{+}-1\right) /(1-$ $r$ ), so that the overall recurrent excitatory synaptic drive in the spontaneous state remains constant as $w_{+}$is varied (Brunel and Wang 2001). Neurons in the inhibitory population are mutually connected with an intermediate weight $w=1$. They are also connected with all

\footnotetext{
${ }^{1}$ The online version of this article contains supplemental data.
}

excitatory neurons in the same layer with the same intermediate weight, which for excitatory-to-inhibitory connections is $w=1$ and for inhibitory-to-excitatory connections is denoted by a weight $w_{\mathrm{I}}$. Neurons in a specific excitatory population are connected to neurons in the nonselective population in the same layer with a feedforward synaptic weight $w=1$ and a feedback synaptic connection of weight $w_{-}$. These parameters were chosen so that in the absence of noise, the spontaneous state, and each of the decision states, were all stable states, a situation we refer to as multistability (Deco and Rolls 2006; Rolls and Deco 2010).

Each individual population is driven by two different kinds of input. First, all neurons in the model network receive spontaneous background activity from outside the module through $N_{\text {ext }}=800$ external excitatory connections. Each synaptic connection carries a Poisson spike train at a spontaneous rate of $3 \mathrm{~Hz}$, which is a typical spontaneous firing rate value observed in the cerebral cortex. This results in a background external input summed over all 800 synapses of 2,400 $\mathrm{Hz}$ for each neuron. Second, the neurons in the two specific populations additionally receive added firing to the external inputs that encode the evidence for the decision to be made. When stimulating, the rate of the Poisson train to the neurons of the specific population D1 is increased by an extra value of $\lambda_{1}$, and to population D2 by $\lambda_{2}$, as these encode the two stimuli to be compared.

We note that in sequential decision-making tasks, used extensively for neurophysiological investigations (Deco et al. 2009; Machens et al. 2005; Rolls and Deco 2010; Romo et al. 2004), and therefore to facilitate comparison used in the fMRI investigation described here, there must be a short-term memory of the first stimulus. This is found neurophysiologically to be present in decision-related systems in the brain (Romo et al. 2004) with the mechanisms for the short-term memory including attractor-related neuronal firing (Machens et al. 2005; Miller and Wang 2006; Rolls 2008) and synaptic facilitation (Deco et al. 2010). It is not the purpose of the present research to model this delay-related activity, because the mechanisms for this have to be present, are a separate issue involving separate mechanisms that have been considered and modeled elsewhere (Deco et al. 2010; Rolls 2008), and because in the present research, the aim was to address the issue of the operation of decision-making circuitry in the brain on correct versus error trials. The modeling results we describe predict the fMRI results found without any need for further hypotheses or assumptions about what might be happening in the delay period. The predictions are about the activity in the decision-making network and reflect changes in activity in this network occurring at the time that information about both decision cues is available. The predictions are thus about the decision-making process itself and apply to situations where the two decision cues are present simultaneously as well as to the sequential design we used in the present fMRI investigation. Predictions were made only about the activity on the decision-making trials and covered a range of values for the differences $\Delta I$ in the magnitude of the two decision stimuli $\lambda_{1}$ and $\lambda_{2}$. We note that in the fMRI investigation described here, the stimulus used for the first cue was randomized across trials and thus would not have contributed differentially on average across trials to the change in the fMRI BOLD signal at the time that the second stimulus was applied, which was when the decision was taken. The simulations were therefore run for $2 \mathrm{~s}$ of spontaneous activity and then for a further $2 \mathrm{~s}$ while the decision stimuli were being applied. During the spontaneous period, the stimuli applied to D1 and D2 (and to all the other neurons in the network) had a value of $3 \mathrm{~Hz}$. (This $3 \mathrm{~Hz}$ is the firing rate being applied by Poisson spikes to all 800 external synaptic inputs of each neuron in D1, so the total synaptic bombardment on each neuron is 2,400 spike/s.) During the decision period, the mean input to D1 and D2 was increased to $3.04 \mathrm{~Hz}$ per synapse (an extra 32 $\mathrm{Hz}$ per neuron). For $\Delta I=0,32$ extra Hertz to the spontaneous was thus applied to each neuron of both D1 and D2. For $\Delta I=16,32+$ $8 \mathrm{~Hz}$ was the extra applied to D1 and corresponds to $\lambda_{1}$ in Fig. 1, and $32-8 \mathrm{~Hz}$ was the extra applied to D2, etc. D1 is thus the correct attractor 
for the decision. The firing rates, and the absolute value of the sum of the synaptic currents (AMPA, NMDA, and GABA, defined in the supplementary material), in all four populations of neurons were saved every $50 \mathrm{~ms}$ for later analysis. The criterion for which population won, that is for which decision was taken, was a mean rate for the last second of the simulation that was $10 \mathrm{~Hz}$ greater than that of the other population. (This is in the context that the spontaneous rate was several spikes/s and that the winning population typically had a mean firing rate of $35-40 \mathrm{~Hz}$ as will be shown.) If the winning population was D1, it was a correct trial, and if the winning population was D2, it was an incorrect trial. This criterion ensured that a stable decision had been reached with a clear winner that stably maintained its high firing rate over a $1 \mathrm{~s}$ period. The criterion was appropriate in that we wished to predict the fMRI BOLD signal that would be obtained on correct versus error trials, and the BOLD signal will be generated by the average of the activity over a period not by a chance high firing rate for $50 \mathrm{~ms}$ that might be obtained due to the noise in the network. For trials on which the decision was correct or incorrect by this criterion, the latency of a decision on that trial was measured by the time of the first $50 \mathrm{~ms}$ bin of three consecutive bins at which the mean rate of the winning population was more than $25 \mathrm{~Hz}$ higher than that of the other population. (These criteria ensure that a decision has been stably reached by using a criterion over the last $1 \mathrm{~s}$ of the simulation and that the decision time is accurately measured given the stochastic nature of the neuronal firing illustrated in Fig. 2, by using just three 50 ms bins over which to estimate when the winning population makes a transition and then using the criterion of $25 \mathrm{~Hz}$ to allow for the variations that occur in the neuronal firing over short time periods of in this case the $150 \mathrm{~ms}$ period in which the latency of the transition was being measured.)
Correct trials
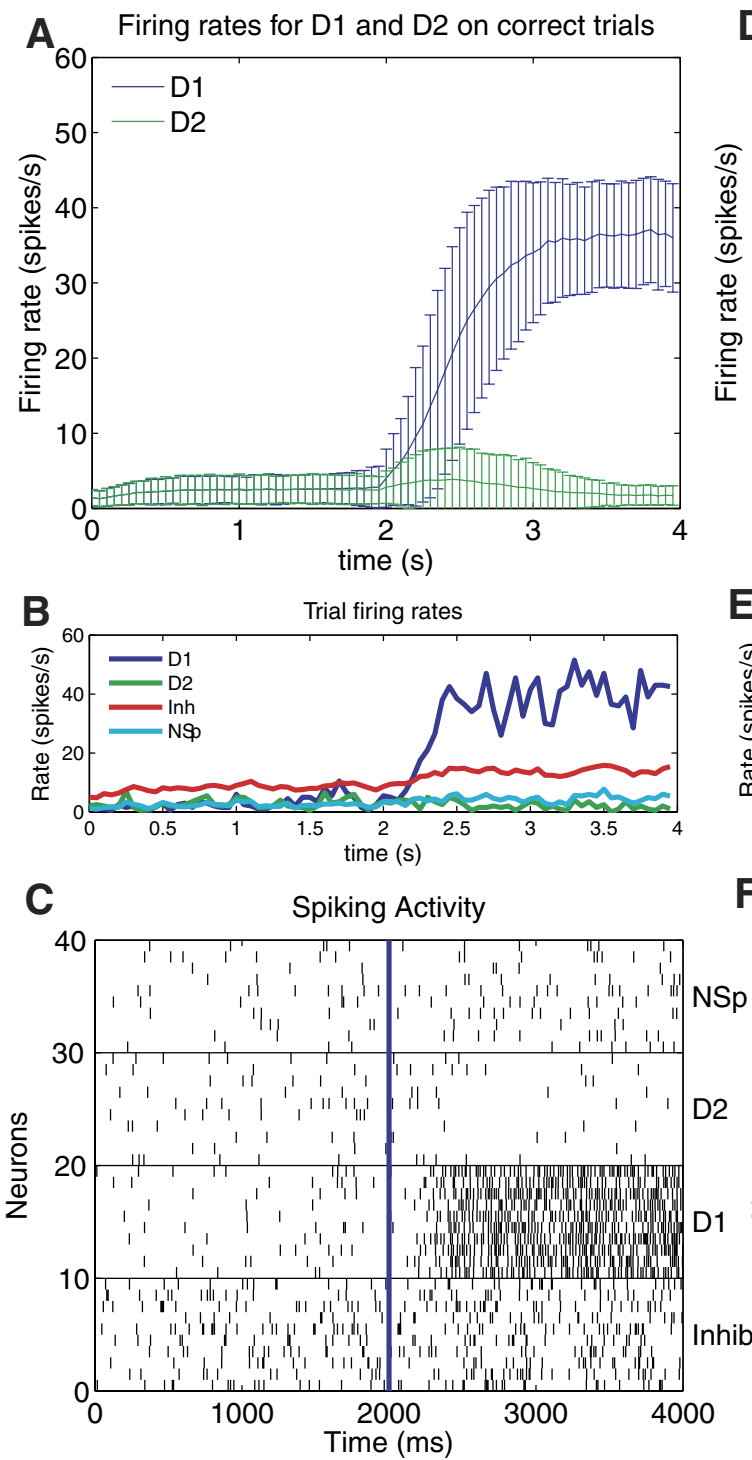

Error trials

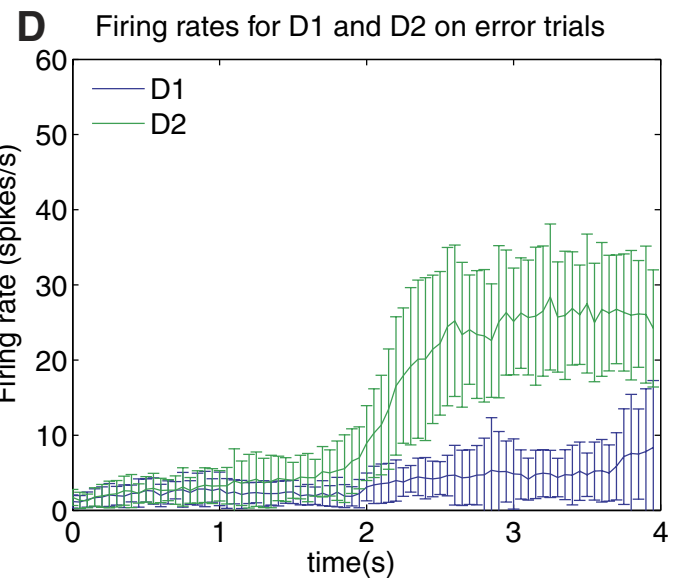

E

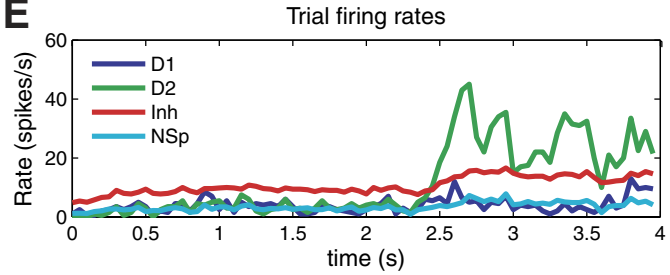

$\mathbf{F}$

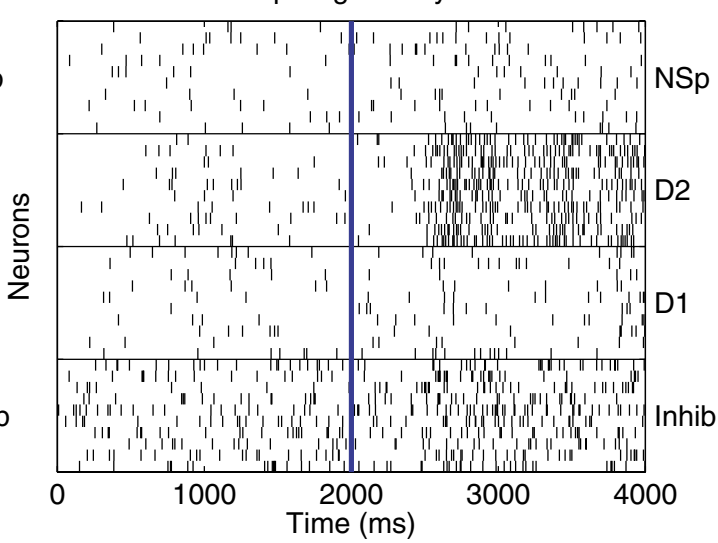

FIG. 2. $A$ and $D$ : firing rates (mean $\pm \mathrm{SD}$ ) for correct and error trials for an intermediate level of difficulty $(\Delta I=32)$. The period $0-2 \mathrm{~s}$ is the spontaneous firing, and the decision cues were turned on at time $=2 \mathrm{~s}$. The means were calculated over 1,000 trials. D1, firing rate of the D1 population of neurons, which is the correct population. D2, firing rate of the D2 population of neurons, which is the incorrect population. A correct trial was one in which the mean rate of the D1 attractor averaged $>10$ spike/s than that of the competing population for the last 1,000 ms of the simulation runs. (Given the attractor nature of the network and the parameters used, the network reached one of the attractors on $>90 \%$ of the 1,000 trials, and this criterion clearly separated these trials as indicated by the mean rates and SDs for the last second of the simulation as shown.) $B$ and $E$ : the firing rates of the 4 populations of neurons on a single trial for a correct $(B)$ and error $(E)$ decision. Inh, the inhibitory population that uses GABA as a transmitter; NSp, the nonspecific population of neurons (see Fig. 1). $C$ and $F$ : rastergrams for the trials shown in $B$ and $E$. 10 neurons from each of the 4 pools of neurons are shown. 
The hemodynamic signal associated with the decision was calculated by convolving the neuronal activity or the synaptic currents of the neurons with the hemodynamic response function used by SPM5 (Statistical Parametric Mapping, Wellcome Trust Centre for Neuroimaging, London) (as this was the function also used in the analyses with SPM of the experimental fMRI data). For the convolution, the predecision period of spontaneous activity was padded out so that it lasted for $30 \mathrm{~s}$, and after the $2 \mathrm{~s}$ period of decision-making activity, each trial was padded out for a further $18 \mathrm{~s}$ with spontaneous activity so that the effects found in a $2 \mathrm{~s}$ period of decision-making could be measured against a steady background. The predicted BOLD signals are shown with $t=0$ corresponding to the time when the decision stimuli were turned on, just as in the analyses of the experimental fMRI data, to enable direct comparisons. [Qualitatively similar results, though with a somewhat slower time course, were obtained with a biophysically based balloon model for the generation of fMRI BOLD signals (Stephan et al. 2007).]

The parameters for the synaptic weights and input currents were chosen using the mean-field equivalent of this network (Brunel and Wang 2001; Deco and Rolls 2006) so that in the absence of noise when the input stimuli are being applied, there were three stable states, the spontaneous firing rate state (with a mean firing for the pyramidal cells of $\sim 3$ spike/s) and two high firing rate attractor states (with a mean firing for the pyramidal cells of $\sim 40$ spike/s), with one neuronal population (D1) representing decision 1 and the other population (D2) decision 2. In particular, $w_{+}$was set to 2.1 .

This multistable regime is one with many interesting decisionmaking properties, including implementing Weber's law $(\Delta I / I=k$, i.e., the change of intensity $I$ that can be reliably detected divided by the intensity value has a linear component) (Deco and Rolls 2006; Deco et al. 2009). With operation in this regime, it is the approximately random spiking times of the neurons (i.e., approximately Poisson firing at a given mean rate) that causes statistical fluctuations that makes the network jump from the spontaneous firing state into one of the high firing rate attractor (decision) states. The randomness of the firing dynamically and probabilistically provokes transitions from the spontaneous firing state to one of the high firing rate attractor basins that represent a decision (see Fig. 1B). The stability of an attractor is characterized by the average time in which the system stays in the basin of attraction under the influence of noise. The noise provokes transitions to other attractor states. It results from the interplay between the Poissonian character of the spikes and the finite-size effect due to the limited numbers of neurons in the network (Rolls and Deco 2010). The theoretical approach and model we use is thus one that has become established as a neuronally realistic model of decision-making (Deco and Rolls 2006; Deco et al. 2009; Rolls and Deco 2010; Wang 2002, 2008). The work we describe in this paper with the model is new in that it extends the analysis of neuronal firing rates on correct and error trials (Wang 2002) to the multistability regime, in which noise plays a large part in the decision-making as the noise is required to provoke the transition to a decision-state and in that we then go on to use the model to make predictions about the fMRI BOLD response that would be obtained from this decisionmaking circuitry on correct versus error trials. The whole analysis of the fMRI data with this perspective on correct versus error trials as a function of $\Delta I$ is also completely new and is an important extension from previous work (Rolls et al. 2010a) in which only overall neuronal activity and the BOLD signal were predicted and analyzed independently of whether a trial was correct or in error. The extension of the approach to error versus correct trials is an important new test and validation of the model and of this approach to how decisionmaking is implemented in the brain.

\section{Olfactory pleasantness decision task}

The design is based on the sequential vibrotactile decision-making studies of Romo and colleagues in which vibrotactile stimuli sepa- rated by a delay were presented, and a decision had to be made when the second stimulus was presented of whether the second frequency was higher than the first (Romo et al. 2004). In our design, the decision was about whether the second odor was more pleasant than the first, or on other trials was more intense than the first. To allow a comparison with trials on which choices between stimuli were not made, there were also trials in which only ratings of the continuous affective value and intensity were made without a choice between stimuli as described in detail by Rolls et al. (2010b). The odors were delivered through a computer-controlled olfactometer. The pleasant odors were $1 \mathrm{M}$ citral and $4 \mathrm{M}$ vanillin. The unpleasant odors were hexanoic acid (10\% vol/vol) and isovaleric acid (15\%). The different trial types and odors were presented in random permuted sequence to control for order effects. We note that this is a variant of the task used by Romo and colleagues (2004), that we are investigating the brain mechanisms for decisions about the pleasantness of stimuli, and that the brain regions activated in these tasks may be different (see DISCUSSION) although we believe that the neuronal mechanisms are similar (Rolls and Deco 2010).

The experimental protocol illustrated in Fig. $1 C$ consisted of an event-related interleaved design presenting in random permuted sequence the three experimental conditions and different pairs of olfactory stimuli for each condition. Each trial started at $t=0 \mathrm{~s}$ with the first odor being delivered for $2 \mathrm{~s}$ accompanied by a visual label stating "sniff first stimulus." There was then a $6 \mathrm{~s}$ period during which clean air was delivered. In this period at $t=7 \mathrm{~s}$, a visual label was displayed stating either "decide pleasantness," "decide intensity," or "rate stimulus." At $t=8 \mathrm{~s}$, the second odor was presented for $2 \mathrm{~s}$ accompanied by a visual label stating either "sniff decide pleasantness," "sniff decide intensity," or "sniff rate stimulus." There was then a $6 \mathrm{~s}$ period during which clean air was delivered. These task instructions, and the presentation of the second odor for a period limited to $2 \mathrm{~s}$, was part of the experimental design to encourage participants to take the decision while the second odor was being presented. Starting at $t=16 \mathrm{~s}$ on decision trials, the words "first stimulus" and "second stimulus" appeared on the screen for $2 \mathrm{~s}$, and in this period, the participant had to select which button key response to make (up or down button) for the decision that had been taken at the time when the second odor was delivered. At $t=18 \mathrm{~s}$ on decision trials, the words "first stimulus" or "second stimulus" were then displayed to provide feedback to the participant that their choice was acknowledged. On rating trials, starting at $t=16 \mathrm{~s}$ the subjective ratings were made. The first rating was for the pleasantness of the second odor on a continuous visual scale from -2 (very unpleasant), through 0 (neutral), to +2 (very pleasant). The second rating was for the intensity of the second odor on a scale from 0 (very weak) to 4 (very intense). The ratings were made with a visual rating scale in which the subject moved the bar to the appropriate point on the scale using a button box. There was $4 \mathrm{~s}$ for each rating. Each of the trial types was presented in random permuted sequence 36 times. Which two of the four odors were presented on each trial, and the order in which they were presented was determined by a random permuted sequence. The onset time for the fMRI analyses described here was at the time of onset of the second odor, which was when the choice decisions were being taken. The fMRI data from the pleasantness choice trials only were used in the analyses described here.

$\Delta I$, the difference in pleasantness of the two stimuli between which a decision was being made, was obtained for each trial by the absolute value of the difference in the (average) rated pleasantness of that pair of stimuli for each subject. These subjective pleasantness ratings were made on different trials from the decision trials for the identical odors during the experimental session as described in more detail elsewhere (Rolls et al. 2010b). The rating of the pleasantness of an odor was made on each rating trial on a visual analog rating scale labeled at one end +2 very pleasant and at the other end -2 very unpleasant. Thus two odors of similar pleasantness would have a small $\Delta I$, and two odors of different pleasantness would have a large $\Delta I$. This measure 
thus reflects the difficulty of the decision and is independent of whether the second odor happened to be pleasant or unpleasant.

Behavioral results for the ratings and the decisions are provided next [and in more detail elsewhere (Rolls et al. 2010b)] to illustrate and validate these procedures and the results. The affective (pleasantness) ratings of the odor stimuli across subjects were (on the scale of $+2=$ very pleasant to $-2=$ very unpleasant) for citral $1.1 \pm 0.1$ (SE), for vanillin $1.2 \pm 0.1$, for hexanoic acid $-0.9 \pm 0.2$, and for isovaleric acid $-0.5 \pm 0.2[F(3,44)=46.3 P \ll 0.001]$. Consistent with these ratings, when making pleasantness decisions, the subjects chose on average the citral $68.4 \pm 11.4 \%$ of the time over the other odors, the vanillin $85.2 \pm 6.1 \%$ of the time, the hexanoic acid $17.2 \pm 7.9 \%$ of the time, and the isovaleric acid $29.2 \pm 10.9 \%$ of the time.

The trials were separated into correct and error trials, and contrasts for correct versus error trials, and additional analyses, were computed with SPM5. Error trials were defined as choices that did not reflect the mean difference in the pleasantness ratings. (The rationale for this was that noise in the system might influence the binary choice of pleasantness made on an individual trial, as predicted by the model, and each participant's estimate of what was the more pleasant of each pair, the input to the decision-making process that corresponds to $\Delta I$, was given by the mean pleasantness rating across all trials for each odor being compared on a particular trial.) For example, if citral was rated on average for pleasantness as +1.5 and vanillin as +1.0 , and the subject chose vanillin over citral, this was classified as an error trial. At the single subject level, contrasts between correct versus error trials were computed within each odor pair. At the group level, these odor-specific contrast maps were combined across subjects. Only contrasts with at least two trials in the error category for each odor pair were included (e.g., 2 error vs. 7 correct trials). Using this criterion, 19 contrast maps from 12 subjects were entered into the group analysis. The results for the main regions did not change when a criterion of at least three trials was used. The mean number of correct trials for each subject was 5.5 for each odor pair, and the mean number of error trials was 3.2 for each odor pair, and there were in total 127 correct and 52 error trials for the SPM regression and contrast analysis and the regression plots.

\section{fMRI data acquisition}

Images were acquired with a 3.0-T VARIAN/SIEMENS wholebody scanner at the Centre for Functional Magnetic Resonance Imaging at Oxford (FMRIB), where $27 \mathrm{~T} 2 *$ weighted EPI coronal slices with in-plane resolution of $3 \times 3 \mathrm{~mm}$ and between plane spacing of $4 \mathrm{~mm}$ were acquired every $2 \mathrm{~s}(\mathrm{TR}=2)$ as described previously for this dataset (Rolls et al. 2010b). Twelve healthy volunteers ( 7 male and 5 female, mean age: 27 ) participated in data collection using olfactory decision-making that was designed to address a number of different issues, including those described here and others described elsewhere (Rolls et al. 2010b). The study was conducted in accord with the declaration of Helsinki and approved by the Central Oxford Research Ethics Committee, and written informed consent from all subjects was obtained before the experiment.

\section{fMRI analyses}

The imaging data were analyzed using SPM5 (Statistical Parametric Mapping, Wellcome Institute of Cognitive Neurology, London). Preprocessing of the data used SPM5 realignment, reslicing with sinc interpolation, normalization to the Montreal Neurological Institute (MNI) coordinate system (Collins et al. 1994), and spatial smoothing with a $6 \mathrm{~mm}$ full width at half-maximum isotropic Gaussian kernel. The time series at each voxel were low-pass filtered with a hemodynamic response kernel. Time series nonsphericity at each voxel was estimated and corrected for (Friston et al. 2002), and a high-pass filter with a cut-off period of $128 \mathrm{~s}$ was applied. In the single event design, a general linear model was then applied to the time course of activation where the decision or rating period onsets $(t=8 \mathrm{~s}$ in each trial) were modeled as single impulse response functions and then convolved with the canonical hemodynamic response function (Friston et al. 1994). Linear contrasts were defined to test specific effects. Time derivatives were included in the basis functions set. Given that the fMRI analyses were to test hypotheses generated by our computational model of decision-related activity in the brain, the main test performed was for brain areas where activations were greater on correct compared with error trials, and brain areas identified in this way were then compared with brain areas implicated by other analyses in making choices. In this hypothesis-led research, we were able to find activations consistent with our hypotheses and to show that such areas are implicated by other analyses in making the choices. Time derivatives were included in the basis functions set. Following smoothness estimation (Kiebel et al. 1999), in the first stage of analysis, condition-specific experimental effects (parameter estimates, or regression coefficients, pertaining to the height of the canonical HRF) were obtained via the general linear model (GLM) in a voxelwise manner for each subject. In the second (group random effects) stage, subject-specific linear contrasts of these parameter estimates were entered into a series of one-sample $t$-test, each constituting a group-level statistical parametric map. We report results for brain regions where there were prior hypotheses about brain areas involved in decision-making or rating affective value including the medial prefrontal cortex and an area to which it is connected the subgenual cingulate cortex, the posterior cingulate cortex, and the dorsolateral prefrontal cortex (Grabenhorst et al. 2008; Hayden et al. 2008; Heekeren et al. 2004; Johansen-Berg et al. 2008; Kable and Glimcher 2007; Kim and Shadlen 1999; McCoy and Platt 2005; Preuschhof et al. 2006; Rolls et al. 2010b) and applied small volume (false discovery rate) corrections for multiple comparisons (Genovese et al. 2002) with a radius corresponding to the full width at half-maximum of the spatial smoothing filter used. Peaks are reported for which $P<0.05$, although the exact corrected probability values (Worsley et al. 1996) are given in the text.

The results were obtained in a GLM including the following regressors: regressors for the first odor presented on every trial; regressors for the second odor presented on every trial (with separate regressors for the 4 different odors, and separate regressors for correct and error trials); for the different regressors modeling a specific second odor on correct and error trials, $\Delta I$ was included as a subject-specific parametric modulator; regressors of the button box responses made during the response period; and regressors for other trial events of no interest. In the second (group random-effects) stage, subject-specific linear contrasts of these parameter estimates were entered into a series of one-sample $t$-test, each constituting a group-level statistical parametric map.

The plots showing correlations between percentage BOLD and $\Delta I$ in Fig. 6 were produced for each subject by taking the average of the BOLD response (in \% BOLD signal change) in the three time bins at 4,6 , and $8 \mathrm{~s}$ poststimulus, on each trial, and the corresponding $\Delta I$ value. The voxels used for extracting BOLD signals were the peak voxels for the correlation with $\Delta I$ found in individual subjects. These were localized by drawing a $6 \mathrm{~mm}$ sphere around the group peak voxel and then localizing the individual subject's peak within that sphere. For each subject, the means were calculated in discretized ranges of delta I (e.g., $0-0.25,0.25-0.5$ etc.), and then these values were averaged across subjects. The time-course graphs in Fig. 5 were created by performing a finite impulse response (FIR) analysis as implemented in SPM5 to make no assumption about the time course based on the temporal filtering property of the hemodynamic response function. The single-subject time courses were then averaged across subjects. 


\section{R E S ULTS}

\section{Attractor network model of decision-making and confidence}

We analyzed the attractor network model of decision-making shown in Fig. 1. Figure 2, $A$ and $D$, shows the mean firing rates of the two neuronal populations D1 and D2 for correct trials $(A)$ and error trials $(D)$ for an intermediate level of task difficulty $(\Delta I=32 \mathrm{~Hz}$; where $\Delta I$ is the difference in spike/s summed across all synapses to each neuron between the 2 inputs, $\lambda_{1}$ to population D1, and $\lambda_{2}$ to population D2). In the correct trials, the population D1 won, and in incorrect trials, the population D2 won. Figure 2, $A$ and $D$, shows that the winning population had higher firing rates on correct than on error trials with an example of a single correct trial shown in Fig. 2, $B$ and $C$, and of a single error trial in $E$ and $F$. From Fig. 2, $A$ and $D$, it is clear that the variability of the firing rate is high from trial to trial with the SDs of the mean firing rate calculated in $50 \mathrm{~ms}$ epochs indicated to quantify the variability. This variability is due to the spiking noise in the network, which influences the decision taken, resulting in probabilistic decision-making, and error choices on some trials. The effects evident in Fig. 2 are quantified, and elucidated over a range of values for $\Delta I$, next.
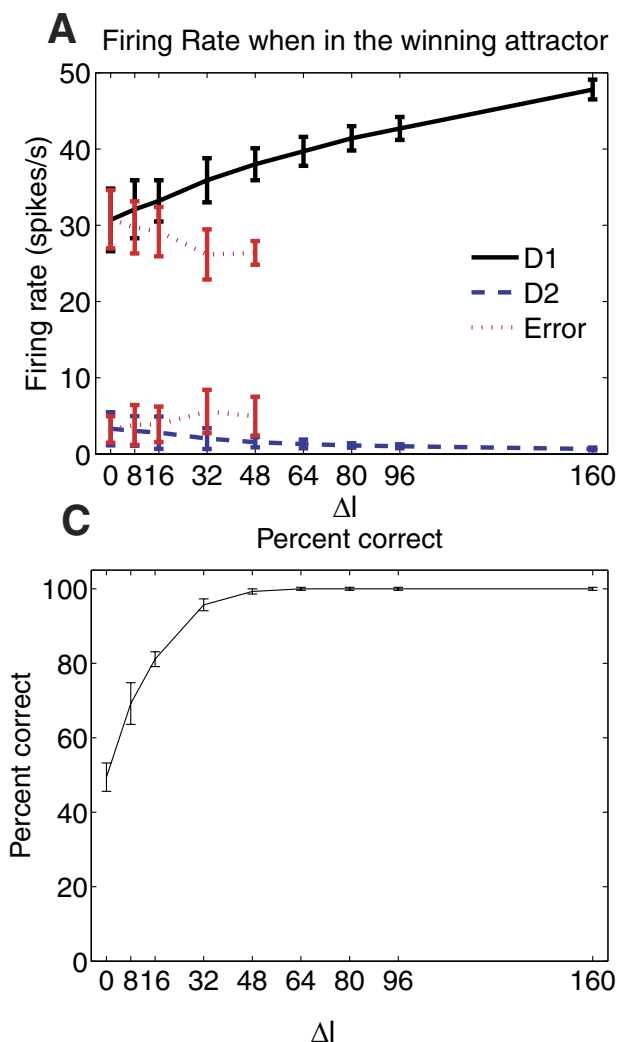

Figure $3 A$ shows the firing rates (mean $\pm \mathrm{SD}$ ) on correct and error trials of the winning and losing attractors as a function of $\Delta I . \Delta I=0$ corresponds to the most difficult decision, and $\Delta I=$ 160 corresponds to easy. It is clear that on correct trials the mean firing rate of the winning attractor D1 increases monotonically as $\Delta I$ increases, and interestingly, the increase is approximately linear (Pearson $r=0.995, P<10^{-6}$ ). The higher mean firing rates of the winning D1 attractor on correct trials as $\Delta I$ increases are due not only to higher peak firing but also to the fact that the variability becomes less as $\Delta I$ increases ( $r=-0.95, P<10^{-4}$ ), reflecting the fact that the system is more noisy and unstable with low $\Delta I$, whereas the firing rate in the correctly winning D1 attractor is maintained more stably with smaller statistical fluctuations against the Poisson effects of the random spike timings at high $\Delta I$. (The measure of variation indicated in the figure is the SD, and this is shown throughout unless otherwise stated to quantify the degree of variation, which is a fundamental aspect of the operation of these neuronal decision-making networks.) The increase of the firing rate when in the D1 attractor on correct trials (solid black line) as $\Delta I$ increases thus reflects the confidence in the decision and, as will be shown next in Fig. $3 C$, the performance as shown by the percentage of correct choices. On correct trials,
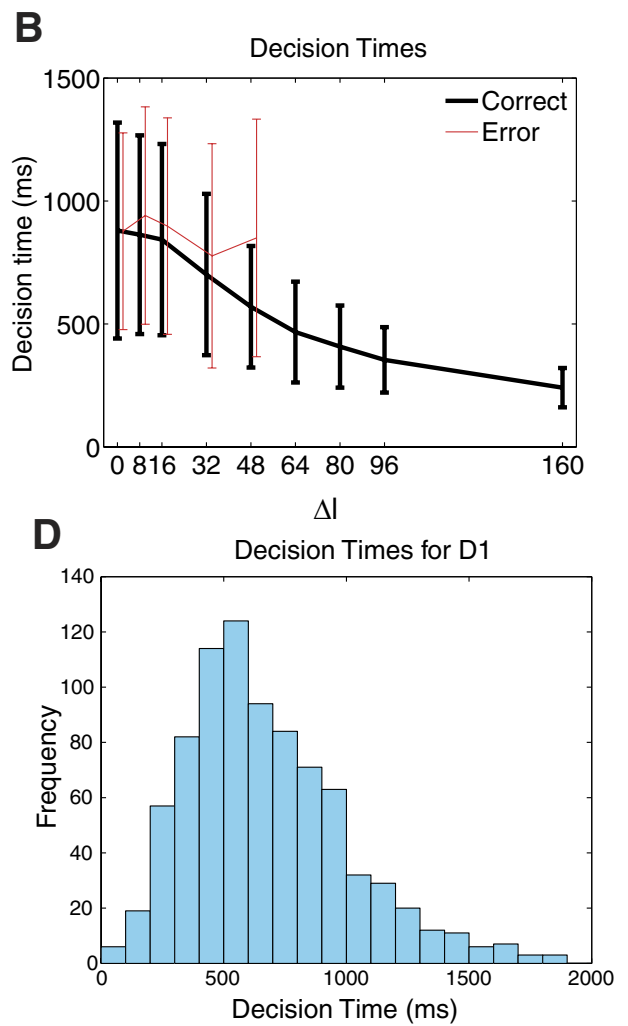

FIG. 3. A: firing rates (mean $\pm \mathrm{SD}$ ) on correct and error trials when in the winning attractor as a function of $\Delta I$. $\Delta I=0$ corresponds to difficult, and $\Delta I=$ 160 corresponds to easy. The firing rates were measured in the last $1 \mathrm{~s}$ of firing, i.e., between $t=3$ and $t=4 \mathrm{~s}$. The firing rates on correct trials for the winning population D1 are shown by solid lines, and for the losing population D2 by dashed lines. All the results are for 1,000 simulation trials for each parameter value, and all the results shown are statistically highly significant. The results on error trials are shown by the dotted red lines, and in this case the D2 attractor wins, and the D1 attractor loses the competition. (There were no error trials for values of $\Delta I=\geqslant 64 \mathrm{~Hz}$.) $B$ : decision or reaction times (mean $\pm \mathrm{SD}$ ) for the D1 population to win on correct trials (thick line), and for D2 to win on error trials (thin line) as a function of the difference in inputs $\Delta I$ to D1 and D2. The plot for the error trials has been offset by a small amount so that its values can be seen clearly. $\Delta I=0$ corresponds to difficult, and $\Delta I=160$ corresponds to easy. $C$ : percent correct performance, i.e., the percentage of trials on which the D1 population won, as a function of the difference in inputs $\Delta I$ to D1 and D2. The mean was calculated over 1,000 trials, and the SD was estimated by the variation in 10 groups each of 100 trials. $\Delta I=0$ corresponds to difficult, and $\Delta I=160$ corresponds to easy. $D$ : the distribution of decision times for the model for $\Delta I=32$ illustrating the long tail of slow responses. Decision times are shown for 837 correct trials, the level of performance was $95.7 \%$ correct, and the mean decision time was $701 \mathrm{~ms}$. 
the firing rate of the losing attractor (D2, dashed blue line) is of course low and decreases further as $\Delta I$ increases on correct trials due to feedback inhibition from the winning D1 attractor, and thus the difference in the firing rates of the two attractors also reflects well the decision confidence.

When we make an incorrect choice, our confidence in our decision is likely to be low (Vickers and Packer 1982). Exactly this is represented in the firing rates of the neurons on incorrect trials, for as shown in dotted red lines in Fig. $3 A$, when an incorrect choice is made (and D2 wins the competition because of noise), the firing rate of the D2 attractor when winning decreases as $\Delta I$ increases (upper dotted red line starting at 31 spike/s). The reason for this is that the external inputs, the evidence for the decision, are now working against the internal recurrent attractor dynamics, which have reached the wrong decision because of the noise in the network. Thus on error trials, the confidence in a decision is also reflected in the firing rates of the attractors.

Conversely the firing rate of the losing attractor (D1) on error trials, which is low with $\Delta I=0(\sim 3$ spike/s $)$, increases by a few spike/second as $\Delta I$ increases (lower dotted red line in Fig. 3A). The reason for this is that the noise has contributed to the D1 attractor losing, and there is more external input against this decision being applied to the D1 neurons as $\Delta I$ increases tending to increase their firing rate from the low value. At the same time, the D1 neurons receive less feedback inhibition from the incorrectly winning population D2 via the inhibitory neurons as $\Delta I$ increases.

The time for the network to reach the correct D1 attractor, i.e., the decision or reaction time of the network, is shown as a function of $\Delta I$ for correct and incorrect trials in Fig. $3 B$ (mean $\pm \mathrm{SD}$ ). Interestingly the decision time continues to decrease $\left(r=-0.95, P<10^{-4}\right)$ over a wide range of $\Delta I$, even when as shown in Fig. $3 C$ the network is starting to perform at $100 \%$ correct. The decreasing decision time as $\Delta I$ increases is attributable to the altered "effective energy landscape" (Rolls and Deco 2010) (see Fig. 1B): a larger input to D1 tends to produce occasionally higher firing rates, and these statistically are more likely to induce a significant depression in the landscape toward which the network flows sooner than with low $\Delta I$. Correspondingly, the variability (quantified by the SD) of the decision times is greatest at low $\Delta I$ and decreases as $\Delta I$ increases $\left(r=-0.95, P<10^{-4}\right)$. This variability would not be found with a deterministic system (i.e., the SDs would be 0 throughout, and such systems include those investigated with mean-field analyses) and is entirely due to the random statistical fluctuations caused by the randomness in the spiking times of the neurons in the integrate-and-fire network.

Very interestingly, the decision times of the network are longer for incorrect (error) than for correct decisions, as shown by the thin graph in Fig. 3B. This difference, though not large, was statistically significant. [The effect was found for the means of the 4 nonzero values of $\Delta I$ for which there were errors $(P<0.05)$, and for $\Delta I=8$ for example, the decision time distribution was longer for error compared with correct trials, $P<0.01$ and confirmed by the nonparametric MannWhitney test.] This models effects that are found in human performance (Vickers and Packer 1982), especially with difficult decisions (Luce 1986; Welford 1980), and that have also been found in lateral intraparietal (LIP) cortex neurons in a motion coherence discrimination task (Roitman and Shadlen
2002) and in a related attractor model of this (Wong et al. 2007). The actual mechanism for this in this biologically plausible attractor decision-making network is that on error trials, the noise is fighting the effects of the difference in the inputs $\lambda_{1}$ and $\lambda_{2}$ that bias the decision-making, and it takes on average relatively long for the noise, on error trials, to become by chance sufficiently large to overcome the effects of this input bias. This effect will contribute to the smaller BOLD signal on error than on correct trials described in the following text. The approach taken here thus provides an account firmly based in the stochastic dynamics of the brain (Rolls and Deco 2010) about how decision or reaction times can be longer on error trials. [Of course there are other reasons why errors may occur, including a failure to maintain attention, distraction, etc, and the reaction times on such trials might be short or long. Slower reaction times on error trials are usually observed in humans when there is no time pressure, while with time pressure the pattern is the opposite (Ratcliff and Rouder 1998). One factor in the short reaction times sometimes found in human performance on error trials may we suggest be a noise-provoked transition from the spontaneous state to a decision state just before or at the time that the decision cues are applied. (This type of error might occur under time pressure because the energy landscape is biased in such a way that the barriers between the states are lower or by effects that increase the noise in the system.) Such trials, in which the spontaneous state was unstable, were excluded from the simulation results described here, although instability of the spontaneous firing state is a subject of considerable but separate interest in relation to the computational processes that may underlie schizophrenia and obsessive-compulsive disorder (Rolls and Deco 2010; Rolls et al. 2008a,b)]. The distribution of decision times for the model has a long tail of slow responses as shown in Fig. $3 D$ for $\Delta I=32$, capturing this characteristic of human reaction time distributions (Luce 1986; Ratcliff et al. 1999; Usher and McClelland 2001; Welford 1980).

At $\Delta I=0$, there is no influence on the network to fall more into attractor D1 representing decision 1 than attractor D2 representing decision 2 , and its decisions are at chance, with $\sim 50 \%$ of decisions being for D1. As $\Delta I$ increases, the proportion of trials on which D1 is reached increases. The relation between $\Delta I$ and percentage correct is shown in Fig. 3C. Interestingly, the performance becomes $100 \%$ correct with $\Delta I=64$, whereas as shown in Fig. $3 A$, the firing rates while in the D1 attractor (and therefore potentially the BOLD signal) continue to increase as $\Delta I$ increases further [and the decision times continue to decrease (Rolls et al. 2010a)] as $\Delta I$ increases further. It is a clear prediction for neurophysiological and behavioral measures that the firing rates with decisions made by this attractor process continue to increase as $\Delta I$ is increased beyond the level for very good performance as indicated by the percentage of correct decisions, and the neuronal and behavioral decision times continue to decrease as $\Delta I$ is increased beyond the level for very good performance. Figure $3 C$ also shows that the variability in the percentage correct (in this case measured over blocks of 100 trials) is large with $\Delta I=0$, and decreases as $\Delta I$ increases. This is consistent with unbiased effects of the noise producing very variable effects in the energy landscape at $\Delta I=0$ and with the external inputs biasing the energy landscape more and more as $\Delta I$ increases, so that 
the flow is much more likely to be toward the D1 attractor (Rolls and Deco 2010).

\section{Predictions of FMRI BOLD signals from the model}

We now show how this model makes predictions for the fMRI BOLD signals that would occur on correct versus incorrect trials in brain areas in which decision-making processing of the type described is taking place. The BOLD signals were predicted from the firing rates of the neurons in the network (or from the synaptic currents flowing in the neurons as described later) by convolving the neuronal activity with the hemodynamic response function in a realistic period, the $2 \mathrm{~s}$ after the decision cues are applied. This is a reasonable period to take as decisions may be taken within this time, and the attractor state may not necessarily be maintained for longer than this. (It is, though, an advantage of this type of model that it is a shortterm memory attractor network that can maintain its firing whether or not the decision cues remain, as this continuing firing enables the decision state to be maintained until a response based on it can be guided and made.) For sequential decision-making tasks, this time corresponds to the $2 \mathrm{~s}$ after the second stimulus is applied, which is the first time at which a decision between the first and second stimuli can be taken.

As shown in Fig. 4A, the predicted fMRI response is larger for correct versus error trials in the decision-making network. The results are shown for a level of difficulty in which there is a reasonable proportion of error trials $(\Delta I=32)$.

Figure $4 B$ shows that the difference predicted from the model between the BOLD signal on correct versus incorrect trials increases with $\Delta I$. The reason for this is that on correct trials with increasing $\Delta I$, the external evidence adds more strongly to the firing produced by the internal recurrent collateral attractor effect in the winning attractor (Fig. $3 A$ ), and the BOLD signal increases with $\Delta I$ (Fig. $4 B$ ). On the other hand, on incorrect trials, the external evidence adds less than on correct trials to the winning attractor and also adds more than on correct trials to the firing rates in the losing attractor, the firing rate of which through the inhibitory interneurons de-

A

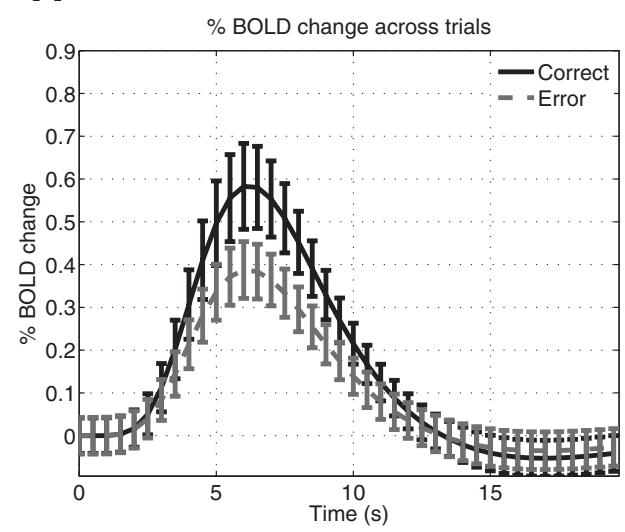

creases the firing in the winning attractor, as shown in Fig. $3 A$. Because on incorrect trials the firing of the winning attractor decreases with $\Delta I$ (Fig. $3 A$ ), the BOLD signal decreases with $\Delta I$ on incorrect trials (Fig. $4 B$ ). An important result of the simulations is that it is the firing rate of the winning attractor that dominates the predicted BOLD signals not the smaller effects seen in the losing attractor. Although it is largely the higher overall neural activity on correct trials that results in the larger predicted BOLD signal on correct than error trials, an additional contributor is the shorter decision latency for correct than error trials illustrated in Fig. $3 B$.

Figure $4 B$ also shows with the thin dotted line (just below the line for the predicted response on correct trials) the predicted response from all trials, that is, correct and error trials. The prediction of the BOLD signal for all trials is relatively close to the prediction for the correct trials because with low $\Delta I$ the predictions for correct and error trials are close, and with larger values of $\Delta I$ there are fewer error trials as indicated in Fig. $3 B$.

\section{fMRI signals that are larger on correct than error trials}

The model predicts that the BOLD signal will be larger in at least some brain areas involved in choice decision-making on correct than on error trials. We tested this by performing a contrast analysis on correct versus incorrect trials in the sequential olfactory decision-making task in which the participants had to decide whether the second odor was more pleasant than the first. Error trials were defined as choices that did not reflect the mean difference in the pleasantness ratings. Four brain regions had significant effects in this correct versus error contrast, and they are the main brain areas implicated by previous studies in value-based decision-making (see METHoDs). They were the posterior cingulate cortex ([ $-18-3234]$ $\mathrm{z}=3.73 P=0.008$ and $[-20-4048] z=3.26 P=0.012)$; the subgenual cingulate cortex $\left(\left[\begin{array}{lll}10 & 24 & -8\end{array}\right] z=3.49 P=\right.$ 0.016); the DLPFC ([24 14 34] $z=3.75 P=0.011$ and [26 56 $36] z=2.99 P=0.037)$; and the medial prefrontal cortex area 10 [(2 $50-12)$ using trials with $\Delta I>1.4$, for which the

\section{B}

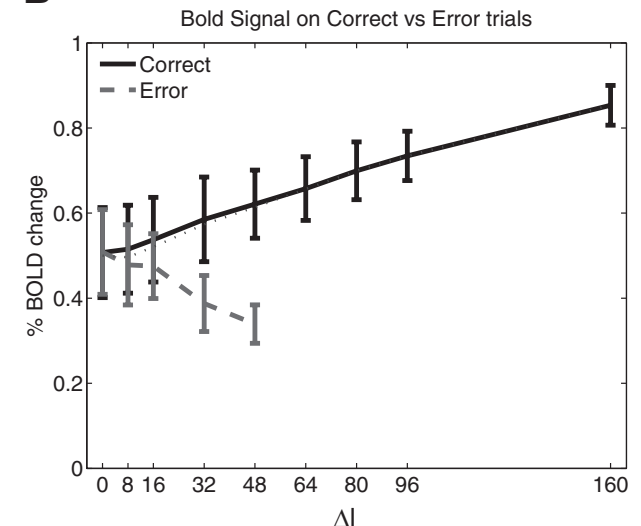

FIG. 4. A: the predicted percentage change (mean $\pm \mathrm{SD}$ ) in the blood-oxygen-level-dependent (BOLD) signal on correct and error trials for $\Delta I=32$. The

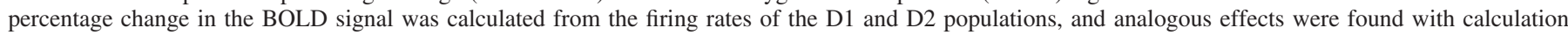

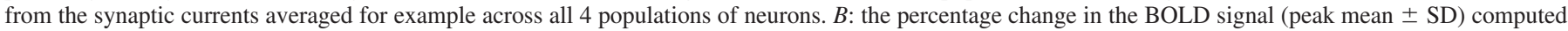

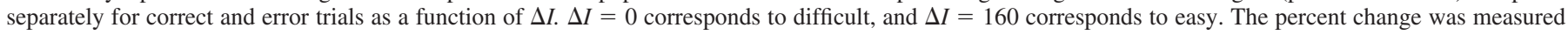

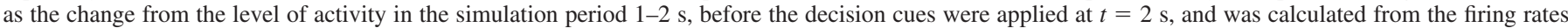

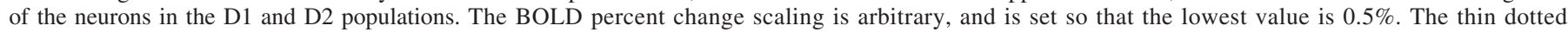
line just below the line for the predicted response on correct trials shows the predicted response from all trials, that is correct and error trials. 
activations were different on correct and error trials with $P<$ $0.05]$. Figure $5 A$ shows the time courses of the activations on correct and on error trials for the DLPFC (left) and subgenual cingulate cortex (right). The brain regions where these activations were found are close to those illustrated in Fig. 6. The parameter estimates associated with these significant effects in these four different brain regions, and in a control brain region, the mid-orbitofrontal cortex, for the correct-error contrast are shown in Fig. $5 B$ (top).
The predictions of the model are that larger activations should be found on correct than on error trials in brain areas implicated in making choices. This prediction was confirmed for the areas described in the preceding text. The model does not make predictions about the exact form of the BOLD response for many factors can influence this. We note that on error trials there was a decrease in the BOLD signal in the medial prefrontal cortex relative to the baseline. It has been noted by others (Summerfield and Koechlin 2008) that in delay
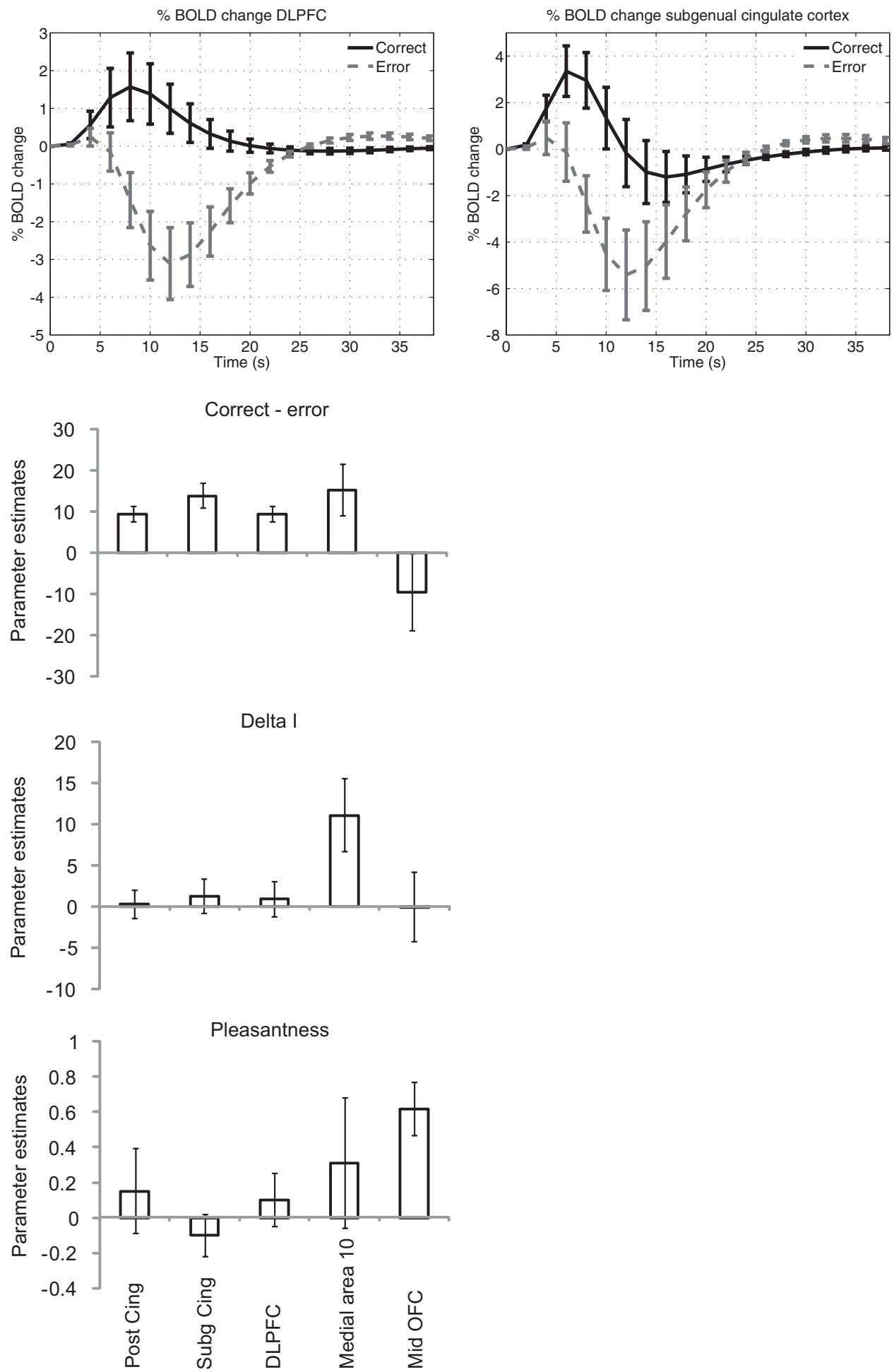

FIG. 5. A: The time courses of the activations on correct and on error trials, for the dorsolateral prefrontal cortex (left) and subgenual cingulate cortex $(r i g h t)$, at sites where the contrast correct-error was significant $B$ : parameter estimates for different brain regions for the correct-error contrast (top), for the correlation of the BOLD signal with $\Delta I$ from all trials (middle), and for the correlation of the BOLD signal with the pleasantness ratings. PostCing, posterior cingulate cortex; SubgCing, subgenual cingulate cortex; DLPFC, dorsolateral prefrontal cortex; Medial area 10, medial prefrontal cortex area 10; MidOFC, midorbitofrontal cortex. (See text for coordinates. The parameter estimates were from 10 participants with $\geq 3$ error trials for each odor pair. 


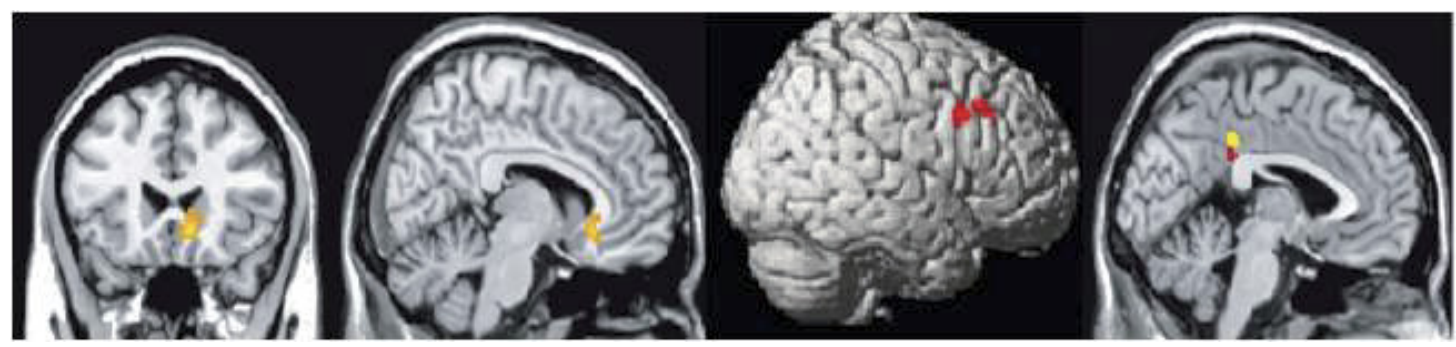

subgenual cingulate cortex

DLPFC

posterior cingulate cortex

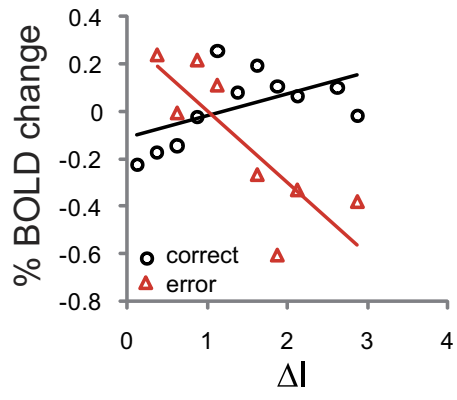

subgenual cingulate cortex

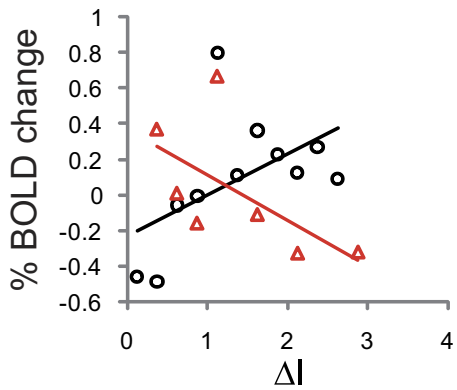

medial area 10

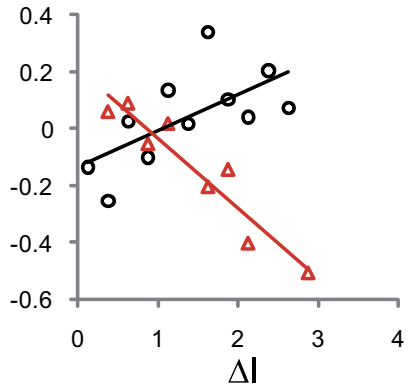

DLPFC

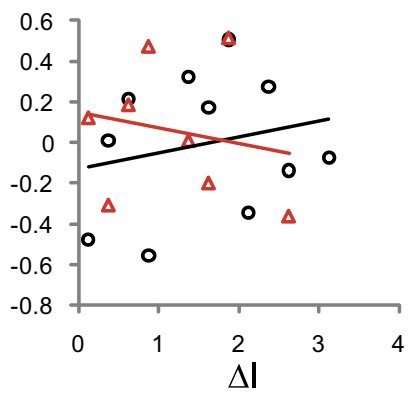

mid OFC

FIG. 6. Top: the subgenual cingulate cortex (with coronal and parasaggittal slices), DLPFC, and posterior cingulate cortex regions with the percentage change in the BOLD signal positively correlated with $\Delta I$ on correct trials and negatively correlated on error trials. Bottom: separate regression plots for the relation between the BOLD signal and $\Delta I$ on correct and on error trials for these regions and for the medial prefrontal cortex area 10, and for a control region, the mid-orbitofrontal cortex in which effects were not found. The regression plots show for discretized values of $\Delta I$ (averaged across subjects) the BOLD signal as a function of $\Delta I$ for correct and incorrect trials. For example, for the posterior cingulate cortex the signs of the correlations $(r=0.51$ on correct trials, and $r=$ -0.91 on error trials) are as predicted for a decision-making area of the brain, and are significantly different $(P<0.001)$ (Baylis 1959$)$. The medial prefrontal cortex coordinates used for extracting the data were $[-454-6]$ with a $10 \mathrm{~mm}$ sphere around this location, the site in the same subjects for the peak of the contrast for easy-difficult trials (Rolls et al. 2010a). The same region [2 50 -12] showed more activity when binary decisions were made compared with when ratings were made in the same subjects (Rolls et al. 2010b).

matching tasks, signals in the ventromedial prefrontal cortex, orbitofrontal cortex, and cingulate cortex are lower during the task than in baseline conditions between trials. Also it is a property that the baseline activation level in some brain regions can be high in the resting state, and against this background, deactivations can be found (Morcom and Fletcher 2007), which may be task-dependent. The important prediction of the model is that the BOLD signal should be larger on correct than on error trials in brain regions involved in making the choice, and this is what was found for the areas described in the preceding text. We go on to show that the magnitude of the difference of the BOLD signal on correct versus error trials increases with $\Delta I$, again as predicted by the model.

To investigate whether correct versus error effects are more likely to be found in areas that are implicated in decisionmaking by an earlier study that showed significant differences for trials on which decisions were made versus trials when only rating and no binary choices were made (Rolls et al. 2010b), we performed ANOVAs on the parameter estimates for correct-incorrect decision contrasts versus having correlations with pleasantness ratings. (In the previous investigation, brain areas implicated in decision-making included prefrontal cortex medial area 10 and in pleasantness ratings, the orbitofrontal cortex.) Figure $5 B$ (bottom) shows the parameter estimates for the correlations of the activations in each of these brain areas with the pleasantness ratings given in every trial to the second odor. It is evident that the posterior cingulate cortex, subgenual cingulate cortex, DLPFC, and medial prefrontal cortex area 10 had considerable parameter estimates for correct-error trials (Fig. 5B, top), and low for correlations with pleasantness (bottom); whereas the orbitofrontal cortex showed high parameter estimates for correlations with pleasantness (bottom) but low for correct-incorrect contrasts (top). ANOVAs on these effects showed a very significant interaction between the factor 
of pleasantness versus correct-incorrect and the factor of brain area $[F(4,36)=6.80, P<0.001]$ with the effect being related to the mid-orbitofrontal cortex in that the interaction effect disappeared when this brain area was removed. Moreover there was a significant interaction between the factor of pleasantness versus correct-incorrect and the factor of brain area: medial area 10 versus mid-orbitofrontal cortex $[F(1,9)=8.00, P=$ 0.02]. Thus activations in the medial prefrontal cortex area 10 , the posterior and subgenual cingulate cortex, and the DLPFC were related more to whether a choice decision was correct or incorrect and less to pleasantness, whereas activations in the mid-orbitofrontal were related more to pleasantness and less to whether the choice was correct or incorrect.

We performed a cross-validation procedure for the main analysis described in Fig. $5 B$ as follows. We used one half of the subject sample to identify a region of interest in a secondlevel SPM contrast analysis and subsequently used the other half of the subject sample for extracting the parameter estimates from the regions identified and used them for the ROI analysis. This analysis confirmed our main findings of a significant difference in correct versus error trials in the posterior cingulate cortex $z=3.69 P=0.009$ and dorsolateral prefrontal cortex $z=2.89, P=0.002$ (uncorrected). The subgenual cingulate cortex effect missed significance due to reduced statistical power resulting from using only half of the subject sample. A subsequent ANOVA on the parameter estimates as described for Fig. $5 B$ (with the subgenual cingulate cortex data removed) showed a significant interaction between the factor of pleasantness versus correct-incorrect and the factor of brain area $[F(3,15)=3.60, P<0.05]$.

\section{fMRI signals linearly related to the correct versus incorrect choices}

Another prediction of the model is that in decision-making areas of the brain, the magnitude of the BOLD signal should increase with $\Delta I$ on correct trials, and decrease with $\Delta I$ on error trials (Fig. 4B). To investigate this, we performed separate SPM regression analyses for correct and error trials in which the regressor for this was $\Delta I$, the mean absolute difference in pleasantness between the two odors presented on a given trial. The analysis was performed at the first (subject) level separately for each second odor. The group second-level analysis was performed across all odors and subjects. We emphasize that in all these cases (the BOLD signal as a function of $\Delta I$ across all trials, across correct trials, and across error trials), the regressor was derived from the model (Fig. 4B).

The analyses showed that for correct trials, positive correlations of the BOLD signal with $\Delta I$ were found in the posterior cingulate cortex ( $[-8-4032] z=3.01 P=0.044$, see Fig. 6). On error trials, negative correlations of the BOLD signal with $\Delta I$ were also found in the posterior cingulate cortex in an area that overlapped (with peaks at $[-20-4052] z=4.10 P=$ 0.001 and $[0-4030] z=4.78 P=0.001)$. These effects are illustrated in Fig. 6. Similar analyses showed similar effects for the subgenual cingulate cortex $\left(\left[\begin{array}{lll}10 & 16 & -4\end{array}\right] z=4.70 P<\right.$ $0.001)$. The signs of the correlations ( $r=0.55$ on correct trials, and $r=-0.81$ on error trials) are as predicted for a decisionmaking area of the brain and are significantly different $(P<$ 0.003). Similar analyses showed similar effects for the dorsolateral prefrontal cortex ([30 18 28 $] z=4.58 P<0.001)$. The signs of the correlations ( $r=0.65$ on correct trials, and $r=$ -0.95 on error trials) are as predicted for a decision-making area of the brain and are significantly different $(P<0.001)$. A positive correlation of the BOLD signal with $\Delta I$ across all trials was found previously in medial prefrontal cortex area 10 (Rolls et al. 2010a), and Fig. 6 shows these effects separated for correct and error trials. The signs of the correlations $(r=0.53$ on correct trials, and $r=-0.62$ on error trials) are as predicted for a decision-making area of the brain and are significantly different $(P=0.032)$.

For comparison, we also show the analyses of the percentage change in the BOLD signal for correct and for error trials for the mid-orbitofrontal cortex, chosen as a control brain region. Activations in this area ([40 36 - 12]) did not have a significant relation to decision-making versus rating (Rolls et al. 2010b) and were not correlated with trial easiness as indexed by $\Delta I$ (Rolls et al. 2010a) but did have a correlation $(z=3.13 P=$ 0.024 ) with the pleasantness of the odor that was presented (Rolls et al. 2010b). The coordinates for this region representing the affective value of the odors but not implicated in the choice decision-making were [40 36 - 12] (Rolls et al. 2010b). In this mid-orbitofrontal cortex region, the correlation between the BOLD signal and $\Delta I$ was $r=0.22$ on correct trials, and $r=$ -0.19 on error trials, and these are not significantly different $(P=0.46)$. Thus this brain area that is not implicated in the binary choice decision-making but does represent information relevant to the decision, the pleasantness of the stimuli, did not have significantly different activations on correct-error trials and did not have significantly different correlations with the BOLD signal on correct and incorrect trials. This is a useful control condition.

Figure $5 B$ also shows (middle) the parameter estimates for the correlation of activations with $\Delta I$ when all trials were included in the regression rather than being separated into correct and error trials. The effects were in general smaller (middle) for the regression of the BOLD signal on $\Delta I$ when all the trials were included, and this is due at least in part to the different effects found on correct and error trials, which tend to cancel each other when all trials are included in the regression. Thus a difference in the correlations with $\Delta I$ for correct versus error trials appears to be a sensitive measure for a brain area involved in decision-making, although as shown in Fig. 5B, a correlation of the BOLD signal with $\Delta I$ using all trials is also likely to be a useful indicator and implicates especially medial prefrontal cortex area 10 in the choice decision-making process. In fact, as shown by the dotted line in Fig. $4 B$, when all trials are combined independently of whether they were correct or error trials, the BOLD signal is expected to increase with $\Delta I$. This is due to the fact that there are fewer error trials as $\Delta I$ increases and that activity in the winning attractor tends to dominate the BOLD signal, which is a conclusion to be made from the model. Thus the high parameter estimate for $\Delta I$ for the medial prefrontal cortex area 10 is an indication that this area is especially involved in the type of decision-making investigated here, choice decisions between different reward values.

An implication is that a sensitive criterion for identifying areas of the brain involved in decision-making is a higher activation on easy than on difficult trials and that this will be especially evident when only correct trials are included in the analysis. A further signature is that the activations are expected 
to increase with $\Delta I$ on correct trials and to decrease on error trials with $\Delta I$. Part of the reason that activations are higher with larger $\Delta I$ with all trials included is that these trials will mostly be correct, whereas difficult trials, with lower values of $\Delta I$, will include more error trials.

\section{I S C U S S I O N}

The spiking network model predicts probabilistic decisionmaking with larger neuronal responses in the winning attractor on correct than on error trials because the spiking noiseinfluenced decision attractor state of the network is consistent with the external evidence, and this neuronal activity in turn predicts larger BOLD responses on correct than error trials in brain regions involved in making choices. Moreover the model predicts that the neuronal activity in the winning attractor and the BOLD response will become larger on correct trials as $\Delta I$ increases and confidence increases and will become smaller in the winning attractor as confidence decreases on error trials as $\Delta I$ increases. If the winner is that for the correct choice, then the firing rate will be higher with increasing $\Delta I$ as then the external evidence, the incoming firing rates, will be higher for this attractor than for the losing attractor, and as $\Delta I$ increases, will increase the firing rates more and more for the winning attractor. On the other hand, if the incorrect attractor wins on a particular trial, then the incoming evidence will provide weak support for the winning (incorrect) attractor and will instead tend to increase the relatively low firing rates of the losing (correct) attractor, which in turn through the inhibitory interneurons will tend to decrease the firing rates further as $\Delta I$ increases in the winning but incorrect attractor. These results of these effects are shown in Fig. 3A.

In this situation, the model is very useful; because it includes synaptic currents and firing rates, it is possible to predict from these the BOLD signal by convolution with the hemodynamic response function. However, on correct trials, as $\Delta I$ increases, the firing rate of the winning (correct) population increases but of the losing (incorrect) population decreases as shown in Fig. $3 A$, so the model is needed to show which effect dominates the BOLD response, and it is found to be the increase in the high firing rate attractor state that dominates the BOLD signal rather than the decrease in the low firing rate state in the losing attractor, as shown in Fig. 4B. Similarly, on incorrect trials as $\Delta I$ increases, the firing rate of the high firing rate incorrect attractor will decrease and of the low firing rate correct attractor will increase, but the net effect predicted for the BOLD signal is a decrease as $\Delta I$ increases (see Fig. $4 B$ ). The model makes qualitatively similar predictions from the synaptic currents, and indeed for this model of cerebral cortical dynamics, the firing rates of the neurons are linearly related to the synaptic currents in the NMDA, AMPA, and GABA receptoractivated ion channels (Rolls and Deco 2010; Rolls et al. 2010a).

We note that predictions about the neuronal firing rates, the synaptic currents though the NMDA, AMPA, and GABA receptor activated ion channels, and thus the BOLD signal, are possible using this type of "mechanistic" model (Rolls and Deco 2010). Any predictions made from the accumulator, counter, or race models of decision-making in which noise influences the accumulation of evidence (Carpenter and Williams 1995; Kiani and Shadlen 2009; Ratcliff et al. 1999;
Smith and Ratcliff 2004; Vickers 1979; Vickers and Packer 1982) are very indirect, as biological mechanisms are not implemented in such models, although there have been extensions to make them more comparable with the underlying biology (Bogacz et al. 2006; Mazurek et al. 2003; Usher and McClelland 2001). Indeed in such accumulator models, a mechanism for computing the difference between the stimuli is not described, whereas in the current model, this is achieved by the feedback inhibition included in the attractor network. Second, in the current model, the decision corresponds to high firing rates in one of the attractors, and there is no arbitrary threshold that must be reached. Third, the noise in the current model is not arbitrary but is accounted for by finite size noise effects of the spiking dynamics of the individual neurons with their Poisson-like spike trains in a system of limited size (Rolls and Deco 2010). Fourth, attractor network models inherently implement a short-term memory by their continuing firing once one attractor has won, and this provides a natural mechanism for maintaining the decision while actions can to taken to implement the decision to obtain the goal (Rolls and Deco 2010). Fifth, although both accumulator and the present integrate-and-fire neuronal attractor network models can be described as implementing a diffusion process when close to the bifurcation (Roxin and Ledberg 2008), the accumulator models are linear, whereas the attractor network model because of the positive feedback in the recurrent connections and the neuronal nonlinearities is nonlinear and captures better nonlinear effects in decision-making (Rolls and Deco 2010). In this context, we note a different approach to decision confidence that involves linear integrators (Moreno-Bote 2010) and also a Bayesian approach that refers to decision confidence (Beck et al. 2008). In contrast, in the present approach, we show how decision confidence is encoded as an emergent property of the operation of the noise-influenced nonlinear decision-making process implemented by an integrate-and-fire attractor network on correct versus error trials as $\Delta I$ changes. Another approach taken to decision-making, in this case about the value of actions, was to surmise that the difference in the value of the two inputs might be a signature of a decision-making process and then to search for voxels in the brain that correlate with this (Wunderlich et al. 2009). In contrast to that approach, the present approach is based on a biologically plausible model of the decision-making process itself and makes clear predictions, tested and confirmed by an fMRI investigation, that the BOLD signal increases with $\Delta I$ in decision-making areas of the brain on correct trials and decreases with $\Delta I$ on error trials as an emergent property of the decision-making process as it occurs in the brain.

The increase of the firing rate of the neurons in the winning attractor with $\Delta I$ on correct trials, and the decrease of the firing rates of the neurons in the winning attractor with $\Delta I$ on error trials is shown here for the first time with the network operating in the multistability regime and echoes what is found when the spontaneous state is not stable when the decision cues are applied (Wang 2002). A new point we now make is that it is a fascinating and important property of this biologically based model of decision-making that decision confidence on correct versus error trials is an emergent property of the neuronal decision-making mechanism in that decision confidence is closely related to $\Delta I$ on correct versus error trials (Petrusic and Baranski 2009; Vickers 1979; Vickers and Packer 1982). 
Neuronal data consistent with this have been recorded by Kiani and Shadlen (2009) in the posterior parietal cortex during a perceptual decision-making task, and neurons in the rat orbitofrontal cortex that respond on correct trials with firing rates that increase with $\Delta I$, that is with confidence (Kepecs et al. 2008), may also be consistent (Insabato et al. 2010; Rolls and Deco 2010). The findings described here that in areas such as the medial prefrontal cortex, posterior and subgenual cingulate cortex, and DLPFC, implicated by other studies in choice decision-making (Boorman et al. 2009; Burgess 2000; Grabenhorst et al. 2008; Hayden et al. 2008; Heekeren et al. 2004; Kim and Shadlen 1999; McCoy and Platt 2005; Preuschhof et al. 2006; Rolls and Grabenhorst 2008; Rolls et al. 2010b), BOLD activations increase with $\Delta I$ and thus confidence on correct trials and decrease on error trials, provide strong support for the approach. [We note that the area of the medial prefrontal cortex that we have identified in this and other studies (Grabenhorst et al. 2008; Rolls and Grabenhorst 2008; Rolls et al. 2010b) as being related to reward-related decisionmaking is in the medial prefrontal cortex area 10 just anterior to the orbitofrontal cortex, and the correspondence between this area and the "orbitofrontal" recording sites in the rat of Kepecs et al. (2008) is not clear.] We also note that the particular brain areas implicated in taking different types of decision are different, even though the underlying mechanism at the local neuronal network level may be similar (Rolls and Deco 2010). For example, in this paper, the fMRI investigation involved choices between rewards in which the ventral medial prefrontal cortex is especially implicated (Grabenhorst et al. 2008; Rolls and Grabenhorst 2008; Rolls et al. 2010a,b), whereas for example choices about the direction of visual motion involve parietal cortex areas (Kiani and Shadlen 2009). The findings also provide a firm theoretical foundation for the use of an increase in the fMRI BOLD signal as decision easiness $(\Delta I)$ increases to identify brain areas involved in decision-making (Heekeren et al. 2004, 2008) but extends that by proposing that an additional signature is that the firing rate in the winning attractor and the BOLD signal increase with (increasing values of) $\Delta I$ on correct trials but decrease with $\Delta I$ on error trials.

An important control point is that activity in the midorbitofrontal cortex that was not implicated in choices between the odors but that did have activations related to the pleasantness of the odors (Rolls et al. 2010b) did not show different activations on correct versus incorrect trials in the present study and did not have activations that increased with $\Delta I$ on correct trials and decreased with $\Delta I$ on error trials and thus were not related to confidence. This is what is predicted for a brain area not involved in binary choice decision-making and its emergent property confidence but in representing pleasantness on a continuous scale, which is likely to be a precursor and prerequisite for choice decisions about pleasantness (Grabenhorst et al. 2008; Rolls and Grabenhorst 2008; Rolls et al. $2010 \mathrm{a}, \mathrm{b})$. We note that $\Delta I$ is not related in any simple way to the pleasantness of the second odor, presented at the onset time for the fMRI analyses described here. Instead $\Delta I$ is the absolute value of the difference in the pleasantness of the first and second odors, and this could be high if the second odor is much less pleasant than the first.

The research described here is the first we know that shows how the firing rates of the neurons in the winning and the losing attractor change approximately linearly with $\Delta I$, i.e., with the easiness of the decision, on correct and on error trials with attractor networks operating in the multistable regime. The research also makes the link to decision confidence, providing evidence that decision confidence is encoded as an emergent property of the operation of the decision-making process on correct versus error trials as $\Delta I$ changes. The research also shows how the decision and hence reaction times are expected to vary with $\Delta I$ on correct and on error trials, provides a clear account of the computational basis of this effect, and indicates that the somewhat longer decision times on error than on correct trials could make some contribution to the smaller fMRI BOLD signal on error trials. The research is also the first to predict that the fMRI BOLD signal will increase approximately linearly with $\Delta I$ in decision-making areas of the brain and decrease approximately linearly with $\Delta I$ on error trials. Moreover, these predictions have a firm and clear theoretical foundation and are fully understandable in terms of the statistical mechanics of the attractor systems implemented in integrate-and-fire networks that we describe (Rolls and Deco 2010). The research is also the first we know to test these predictions in an fMRI investigation and has shown, in brain areas implicated by other evidence in taking the choice decisions, that the BOLD signal does increase on correct trials and decrease on error trials as $\Delta I$ increases. The fMRI investigation and analyses thus provide strong support for this theory of choice decision-making in the brain (Rolls and Deco 2010). Moreover the research goes beyond this by showing that these characteristic signatures of the BOLD signal in a choice decision-making brain system are not found in other areas such as the mid-orbitofrontal cortex in which the continuous-valued reward evidence for the decision is represented (Rolls 2005, 2010), as shown by the correlation of the BOLD signal there with the pleasantness ratings of the stimuli.

Finally, the present work provides a unifying approach (Rolls 2008; Rolls and Deco 2010) to decision-making and decision confidence and to how spiking-related noise in the brain affects choice, confidence, synaptic and neuronal activity, and fMRI signals, and how these relate to correct versus incorrect performance. In this paper, we have investigated choice mechanisms between rewards, and the results implicate especially the medial prefrontal cortex area 10 in this (see also Rolls et al. 2010b). It is suggested that decision-making categorization effects found in other brain areas [area LIP about global visual motion (Churchland et al. 2008; Gold and Shadlen 2002, 2007; Shadlen and Newsome 1996); DLPFCl and ventral premotor cortex about the intensity of odors (Rolls et al. 2010b); the ventral premotor cortex about the frequency of vibrotactile stimuli (Deco et al. 2009; Romo and Salinas 2003)] operate by a similar noise-influenced attractor mechanism that effectively encodes decision confidence (Rolls and Deco 2010). Moreover the approach generalizes to other processes implemented by attractor networks in the brain including memory recall, and indeed we propose that confidence in a recalled memory when the recall is correct or in error is an emergent property of the memory recall process (Rolls 2008; Rolls and Deco 2010) in the same way as that described here. 


\section{A C K N O W LE D G MEN TS}

The fMRI investigation was performed at the Centre for Functional Magnetic Resonance Imaging of the Brain (FMRIB) at Oxford University, and we thank P. Hobden and I. Tracey for help.

Present address of F. Grabenhorst: Dept. of Physiology, Development and Neuroscience, University of Cambridge, UK.

\section{G R A N T S}

F. Grabenhorst was supported by the Gottlieb-Daimler- and Karl BenzFoundation and by the Oxford Centre for Computational Neuroscience. The research was supported by the Oxford Centre for Computational Neuroscience. G. Deco received support from the McDonnell Centre for Cognitive Neuroscience at Oxford University and from grant CONSOLIDER Bilingualism and Cognitive Neuroscience and project BFU (Spanish Ministry of Science).

\section{I S C L OS URES}

No conflicts of interest, financial or otherwise, are declared by the author(s).

\section{REFERENCES}

Abeles M. Corticonics-Neural Circuits of the Cerebral Cortex. New York: Cambridge, 1991.

Baylis NTJ. Statistical Methods in Biology. London: English Universities Press, 1959.

Beck JM, Ma WJ, Kiani R, Hanks T, Churchland AK, Roitman J, Shadlen MN, Latham PE, Pouget A. Probabilistic population codes for Bayesian decision making. Neuron 60: 1142-1152, 2008.

Bogacz R, Brown E, Moehlis J, Holmes P, Cohen JD. The physics of optimal decision making: a formal analysis of models of performance in two-alternative forced-choice tasks. Psychol Rev 113: 700-765, 2006.

Boorman ED, Behrens TE, Woolrich MW, Rushworth MF. How green is the grass on the other side? Frontopolar cortex and the evidence in favor of alternative courses of action. Neuron 62: 733-743, 2009.

Brunel N, Wang XJ. Effects of neuromodulation in a cortical network model of object working memory dominated by recurrent inhibition. J Comput Neurosci 11: 63-85, 2001.

Burgess PW. Strategy application disorder: the role of the frontal lobes in human multitasking. Psychol Res 63: 279-288, 2000.

Carpenter RHS, Williams ML. Neural computation of log likelihood in control of saccadic eye movements. Nature 377: 59-62, 1995.

Churchland AK, Kiani R, Shadlen MN. Decision-making with multiple alternatives. Nat Neurosci 11: 693-702, 2008.

Collins DL, Neelin P, Peters TM, Evans AC. Automatic 3D intersubject registration of MR volumetric data in standardized Talairach space. $J$ Comput Assist Tomogr 18: 192-205, 1994.

Dayan P, Abbott LF. Theoretical Neuroscience. Cambridge, MA: MIT Press, 2001.

Deco G, Rolls ET. Decision-making and Weber's Law: a neurophysiological model. Eur J Neurosci 24: 901-916, 2006.

Deco G, Rolls ET, Romo R. Stochastic dynamics as a principle of brain function. Prog Neurobiol 88: 1-16, 2009.

Deco G, Rolls ET, and Romo R. Synaptic dynamics and decision-making Proc Natl Acad Sci USA 107: 7545-7549, 2010.

Deco G, Scarano L, Soto-Faraco S. Weber's law in decision making: integrating behavioral data in humans with a neurophysiological model. $J$ Neurosci 27: 11192-11200, 2007.

Friston KJ, Glaser DE, Henson RN, Kiebel S, Phillips C, Ashburner J. Classical and Bayesian inference in neuroimaging: applications. NeuroImage 16: 484-512, 2002.

Friston KJ, Worsley KJ, Frackowiak RSJ, Mazziotta JC, Evans AC. Assessing the significance of focal activations using their spatial extent. Hum Brain Map 1: 214-220, 1994.

Genovese CR, Lazar NA, Nichols T. Thresholding of statistical maps in functional neuroimaging using the false discovery rate. NeuroImage 15: 870-878, 2002.

Gold JI, Shadlen MN. Banburismus and the brain: decoding the relationship between sensory stimuli, decisions, and reward. Neuron 36: 299-308, 2002.

Gold JI, Shadlen MN. The neural basis of decision making. Аnпи Rev Neurosci 30: 535-574, 2007.

Grabenhorst F, Rolls ET, Parris BA. From affective value to decisionmaking in the prefrontal cortex. Eur J Neurosci 28: 1930-1939, 2008.
Hayden BY, Nair AC, McCoy AN, Platt ML. Posterior cingulate cortex mediates outcome-contingent allocation of behavior. Neuron 60: 19-25, 2008.

Heekeren HR, Marrett S, Bandettini PA, Ungerleider LG. A general mechanism for perceptual decision-making in the human brain. Nature 431: 859-862, 2004.

Heekeren HR, Marrett S, Ungerleider LG. The neural systems that mediate human perceptual decision making. Nat Rev 9: 467-479, 2008.

Insabato A, Pannunzi M, Rolls ET, Deco G. Confidence-related decisionmaking. J Neurophysiol 104: 539-547, 2010.

Johansen-Berg H, Gutman DA, Behrens TE, Matthews PM, Rushworth MF, Katz E, Lozano AM, Mayberg HS. Anatomical connectivity of the subgenual cingulate region targeted with deep brain stimulation for treatment-resistant depression. Cereb Cortex 18: 1374-1383, 2008.

Jonsson FU, Olsson H, Olsson MJ. Odor emotionality affects the confidence in odor naming. Chem Senses 30: 29-35, 2005.

Kable JW, Glimcher PW. The neural correlates of subjective value during intertemporal choice. Nat Neurosci 10: 1625-1633, 2007.

Kepecs A, Uchida N, Zariwala HA, Mainen ZF. Neural correlates, computation and behavioural impact of decision confidence. Nature 455: 227-231, 2008.

Kiani R, Shadlen MN. Representation of confidence associated with a decision by neurons in the parietal cortex. Science 324: 759-764, 2009.

Kiebel SJ, Poline JB, Friston KJ, Holmes AP, Worsley KJ. Robust smoothness estimation in statistical parametric maps using standardized residuals from the general linear model. Neuroimage 10: 756-766, 1999.

Kim JN, Shadlen MN. Neural correlates of a decision in the dorsolateral prefrontal cortex of the macaque. Nat Neurosci 2: 176-185, 1999.

Luce RD. Response Times: Their Role in Inferring Elementary Mental Operation Organization. New York: Oxford, 1986.

Machens CK, Romo R, Brody CD. Flexible control of mutual inhibition: a neural model of two-interval discrimination. Science 307: 1121-1124, 2005.

Marti D, Deco G, Mattia M, Gigante G, Del Giudice P. A fluctuation-driven mechanism for slow decision processes in reverberant networks. PLOS ONE 3: e2534, 2008.

Mazurek ME, Roitman JD, Ditterich J, Shadlen MN. A role for neural integrators in perceptual decision making. Cereb Cortex 13: 1257-1269, 2003.

McCoy AN, Platt ML. Risk-sensitive neurons in macaque posterior cingulate cortex. Nat Neurosci 8: 1220-1227, 2005.

Miller P, Wang XJ. Inhibitory control by an integral feedback signal in prefrontal cortex: a model of discrimination between sequential stimuli. Proc Natl Acad Sci USA 103: 201-206, 2006.

Morcom AM, Fletcher PC. Does the brain have a baseline? Why we should be resisting a rest. NeuroImage 37: 1073-1082, 2007.

Moreno-Bote R. Decision confidence and uncertainty in diffusion models with partially correlated neuronal integrators. Neural Comput 22: 1786-1811, 2010.

Petrusic WM, Baranski JV. Probability assessment with response times and confidence in perception and knowledge. Acta Psychol 130: 103-114, 2009.

Preuschhof C, Heekeren HR, Taskin B, Schubert T, Villringer A. Neural correlates of vibrotactile working memory in the human brain. $J$ Neurosci 26: 13231-13239, 2006.

Ratcliff R, Rouder JN. Modeling response times for two-choice decisions. Psychol Sci 9: 347-356, 1998.

Ratcliff R, Van Zandt T, McKoon G. Connectionist and diffusion models of reaction time. Psychol Rev 106: 261-300, 1999.

Roitman JD, Shadlen MN. Response of neurons in the lateral intraparietal area during a combined visual discrimination reaction time task. J Neurosci 22: 9475-9489, 2002.

Rolls ET. Emotion Explained. Oxford, UK: Oxford Univ. Press, 2005.

Rolls ET. Memory, Attention, and Decision-Making: A Unifying Computational Neuroscience Approach. Oxford, UK: Oxford Univ. Press, 2008.

Rolls ET. Taste, olfactory, and food texture reward processing in the brain and obesity. Int J Obesity 53: 694-706, 2010a.

Rolls ET, Deco G. Computational Neuroscience of Vision. Oxford, UK: Oxford Univ. Press, 2002.

Rolls ET, Deco G. The Noisy Brain: Stochastic Dynamics as a Principle of Brain Function. Oxford, UK: Oxford Univ. Press, 2010.

Rolls ET, Grabenhorst F. The orbitofrontal cortex and beyond: from affect to decision-making. Prog Neurobiol 86: 216-244, 2008.

Rolls ET, Grabenhorst F, Deco G. Choice, difficulty, and confidence in the brain. NeuroImage; doi:10.1038/ijo.2010.155. 
Rolls ET, Grabenhorst F, Parris BA. Neural systems underlying decisions about affective odors. J Cognit Neurosci 22: 1069-1082, 2010 b.

Rolls ET, Loh M, Deco G. An attractor hypothesis of obsessive-compulsive disorder. Eur J Neurosci 28: 782-793, 2008a.

Rolls ET, Loh M, Deco G, Winterer G. Computational models of schizophrenia and dopamine modulation in the prefrontal cortex. Nat Rev Neurosci 9: 696-709, 2008b.

Romo R, Hernandez A, Zainos A. Neuronal correlates of a perceptual decision in ventral premotor cortex. Neuron 41: 165-173, 2004.

Romo R, Salinas E. Flutter discrimination: neural codes, perception, memory and decision making. Nat Rev Neurosci 4: 203-218, 2003.

Roxin A, Ledberg A. Neurobiological models of two-choice decision making can be reduced to a one-dimensional nonlinear diffusion equation. PLoS Comput Biol 4: e1000046, 2008.

Shadlen MN, Newsome WT. Motion perception: seeing and deciding. Proc Natl Acad Sci USA 93: 628-633, 1996.

Shadlen MN, Newsome WT. Neural basis of a perceptual decision in the parietal cortex (area LIP) of the rhesus monkey. J Neurophysiol 86: 1916-1936, 2001.

Smith PL, Ratcliff R. Psychology and neurobiology of simple decisions. Trends Neurosci 27: 161-168, 2004.

Stephan KE, Weiskopf N, Drysdale PM, Robinson PA, Friston KJ. Comparing hemodynamic models with DCM. Neuroimage 38: 387-401, 2007.
Summerfield C, Koechlin E. A neural representation of prior information during perceptual inference. Neuron 59: 336-347, 2008.

Usher M, McClelland JL. The time course of perceptual choice: the leaky, competing accumulator model. Psychol Rev 108: 550-592, 2001.

Vickers D. Decision Processes in Visual Perception. New York: Academic, 1979.

Vickers D, Packer J. Effects of alternating set for speed or accuracy on response time, accuracy and confidence in a unidimensional discrimination task. Acta Psychol 50: 179-197, 1982.

Wang XJ. Probabilistic decision making by slow reverberation in cortical circuits. Neuron 36: 955-968, 2002.

Wang XJ. Decision making in recurrent neuronal circuits. Neuron 60: 215 234, 2008.

Welford AT, editor, Reaction Times. London: Academic, 1980.

Wong KF, Huk AC, Shadlen MN, Wang XJ. Neural circuit dynamics underlying accumulation of time-varying evidence during perceptual decision making. Front Comput Neurosci 1: 6, 2007.

Worsley KJ, Marrett P, Neelin AC, Friston KJ, Evans AC. A unified statistical approach for determining significant signals in images of cerebral activation. Hum Brain Mapp 4: 58-73, 1996.

Wunderlich K, Rangel A, O'Doherty JP. Neural computations underlying action-based decision making in the human brain. Proc Natl Acad Sci USA 106: 17199-17204, 2009 . 